Ronald P. Muraro

EDIS FE631

Fritz M. Roka

http://edis.ifas.ufl.edu/FE631

Robert E. Rouse

\title{
Budgeting Costs and Returns for Southwest Florida Citrus Production, 2004-05
}

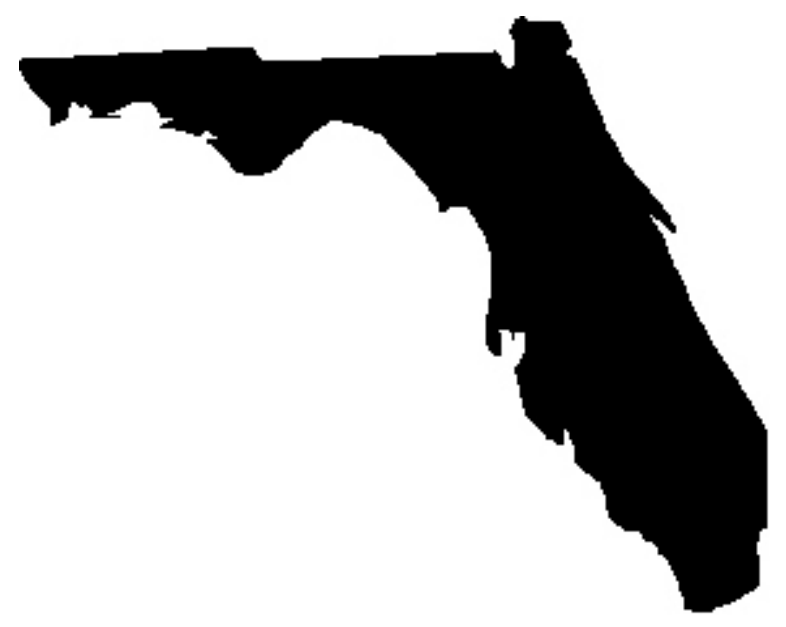

UNIVERSITY OF 10. $\mathrm{ILORIDA}$

\section{IFAS EXTENSION}

Institute of Food and Agricultural Sciences

Food and Resource Economics Department

Florida Agricultural Experiment Station

Florida Cooperative Extension Service 


\title{
ABSTRACT
}

Estimated costs and returns of growing processed-market round oranges and fresh-market seedless grapefruit in the Southwest area of Florida are presented for the tenth consecutive year. The format presented may be used by individual growers to budget costs and returns, utilizing individual data on specific groves.

Key words: citrus, budgeting, costs, round oranges, seedless grapefruit and Southwest Florida.

NOTE: Southwest Florida refers primarily to Charlotte, Collier, Glades, Hendry and Lee counties. However, the costs shown are applicable to other South Central Florida counties such as DeSoto, Okeechobee, and Sarasota counties.

The budgeted cost information presented herein is the most current available. The budget cost items have been revised to reflect current grove practices being used by growers--e.g., chemical mowing, different spray materials and rates of fertilization, microsprinkler irrigation, more reset trees, etc. The 2004-2005 budgets reflect major cost increases in all production inputs: fuel averaged a $22 \%$ increase; fertilizer products increased $15 \%$; chemicals averaged an $8 \%$ increase; and equipment operation costs increased $7 \%$. Along with the increased costs, three major hurricanes (storms) during August and September 2004 resulted in wide tree damage and fruit loss. The Indian River region experienced fruit loss of $70 \%$ to $80 \%$ on red and white grapefruit, respectively. Hamlin orange losses in the Central Florida (ridge) region were $30 \%$ to $40 \%$ with Valencia orange losses between $20 \%$ and $30 \%$. The only citrus growing region that was not significantly affected by the three storms was the Southwest Florida citrus region. As a result of the excessive fruit loss, the per box, per pound solid and per carton costs for the Indian River and Central (ridge) growing regions were substantially higher than in recent years.

The budget costs in this report represent an owner-managed operation for the production of oranges for processing and grapefruit for the fresh market. Therefore, the 10 percent handling and supervision charge added to the material cost for a custom-managed operation is not included in the costs.

\begin{abstract}
Although the estimated annual per acre grove costs listed are representative for a mature citrus grove (10+ years old), the grove care costs for a specific grove site may differ depending upon the tree age, tree density and the grove practices performed; e.g., spot herbicide for grass/brush regrowth under trees could add an additional $\$ 11.88$ per acre; Diaprepes control could add $\$ 84.18$ per acre for each foliar application; extensive tree loss due to blight or tristeza could substantially increase the tree replacement and care costs; spray applications to control citrus leafminer and nematicide applications such as Temik (\$116.94/acre) could increase the total cultural costs per acre above the average costs shown in the comparative budgets; travel and set-up costs may vary due to size of the citrus grove and distance from the grove equipment barn and could add $\$ 28.86$ per acre; etc.
\end{abstract}

\section{ACKNOWLEDGEMENTS}

Appreciation is extended to Mrs. Jane Wilson for typing the final draft of this manuscript.

Appreciation is also extended to the citrus growers and production managers of Southwest Florida who provided suggestions for the revision of this manuscript. 


\section{TABLE OF CONTENTS}

$\underline{\text { Page }}$

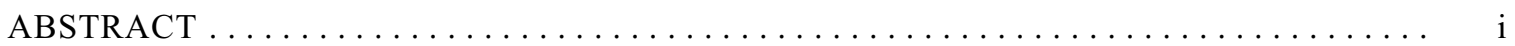

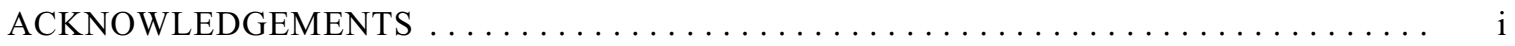

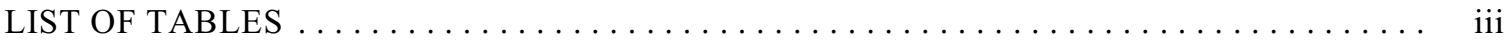

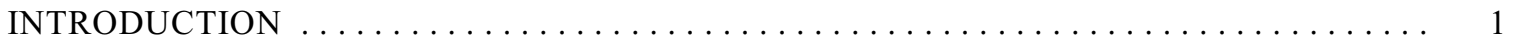

METHOD OF DATA COLLECTION $\ldots \ldots \ldots \ldots \ldots \ldots \ldots \ldots \ldots \ldots \ldots \ldots \ldots \ldots \ldots \ldots \ldots \ldots \ldots$

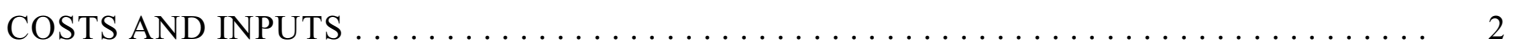

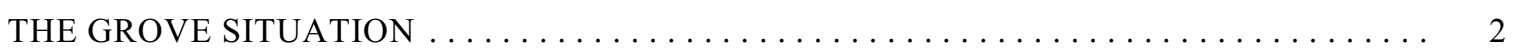

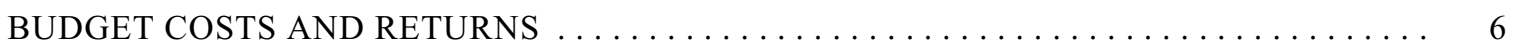

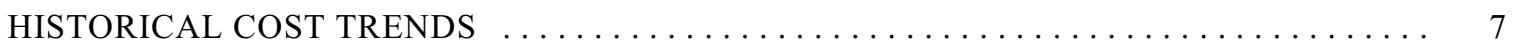

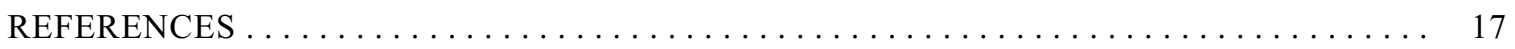

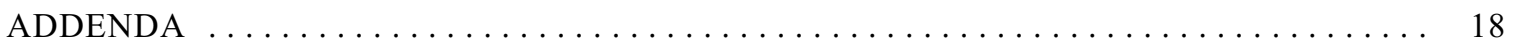

NOTE: The ADDENDA include a Listing of Grove Care Programs for Southwest Florida Citrus Production for Both Round Oranges and Grapefruit; 2005 custom rate summary report; cost of establishing a citrus grove; etc. Page 18 is a list of the tables included in the ADDENDA. 


\section{LIST OF TABLES}

$\underline{\text { Table }}$

Schedule of production practices and budget items for a Southwest Florida mature,

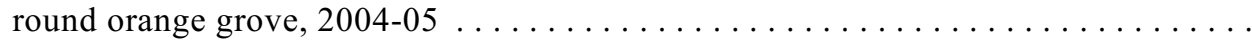

Schedule of production practices and budget items for a Southwest Florida mature, red seedless grapefruit grove, $2004-05 \ldots \ldots \ldots \ldots \ldots \ldots \ldots \ldots \ldots$

Calculation of normal production per tree and per acre for Hamlin oranges, 2004-05

Calculation of normal production per tree and per acre for red seedless grapefruit, 2004-05.

Estimated annual per acre costs and returns for a mature, Hamlin orange grove producing for the processed market, Southwest Florida area, 2004-05 . . . . . . . .

Estimated total delivered-in cost for Southwest Florida Hamlin oranges grown for the processed juice market under three cultural cost programs, 2004-05 . . . . . . . .

Estimated annual per acre costs and returns for a mature, red seedless grapefruit grove producing for the fresh market, Southwest Florida area, 2004-05 . . . . . . . .

Estimated total delivered-in cost for Southwest Florida Red grapefruit under three cultural cost programs, 2004-05

Estimated F.O.B. cost for fresh market Southwest Florida Red grapefruit, 2004-05 . .

Estimated annual per acre costs and returns and 5-year average costs and returns for a mature, Hamlin orange grove producing citrus for processed market in the

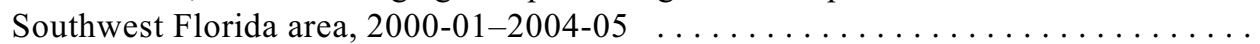

Estimated annual per acre costs and returns and 5-year average costs and returns (adjusted to 2005 dollars) for a mature, Hamlin orange grove producing citrus for processed market in the Southwest Florida area, 2000-01-2004-05 . . . . . . .

Estimated annual per acre costs and returns and 5-year average costs and returns for a mature, red seedless grapefruit grove producing citrus for fresh fruit packing in the

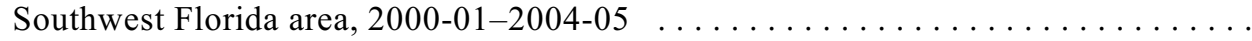

Estimated annual per acre costs and returns and 5-year average costs and returns (adjusted to 2005 dollars) for a mature, red seedless grapefruit grove producing citrus for fresh fruit packing in the Southwest Florida area, 2000-01-2004-05 . . . . 


\title{
BUDGETING COSTS AND RETURNS FOR SOUTHWEST \\ FLORIDA CITRUS PRODUCTION, 2004-05
}

\author{
Ronald P. Muraro, Fritz M. Roka, and Robert E. Rouse
}

INTRODUCTION

Southwest Florida has become a major citrus production area. In 2004 citrus acreage in Southwest Florida represented over $22.6 \%$ of total state citrus acreage. Acreage in Southwest Florida increased from 72,480 in 1986 to 179,948 in 1998 then decreased to 169,386 in 2004 . The $5.9 \%$ decline in acreage was primarily due to trees on sour orange rootstock that died from tristeza virus and acreage destroyed in the citrus canker eradication program. Southwest Florida refers primarily to Charlotte, Collier, Glades, Hendry and Lee counties. However, the costs shown are applicable to other South Central Florida counties such as DeSoto, Okeechobee, and Sarasota counties.

Budget analysis provides the basis for many grower decisions. Budget analysis can be used to calculate potential profits from an operation, to determine cash requirements for an operation, and to determine break-even prices. This report presents two budgets constructed from current data and provides a format for growers to analyze costs and returns from their individual records.

The 2004-2005 budgets reflect major cost increases in all production inputs: fuel averaged a $22 \%$ increase; fertilizer products increased $15 \%$; chemicals averaged an $8 \%$ increase; and equipment operation costs increased 7\%. Along with the increased costs, three major hurricanes (storms) during August and September 2004 resulted in wide tree damage and fruit loss. The Indian River region experienced fruit loss of $70 \%$ to $80 \%$ on red and white grapefruit, respectively. Hamlin orange losses in the Central Florida (ridge) region were $30 \%$ to $40 \%$ with Valencia orange losses between $20 \%$ and $30 \%$. The only citrus growing region that was not significantly affected by the three storms was the Southwest Florida citrus region. As a result of the excessive fruit loss, the per box, per pound solid and per carton costs for the Indian River and Central (ridge) growing regions were substantially higher than in recent years.

\section{METHOD OF DATA COLLECTION}

The data presented here were developed by surveying custom operators, input suppliers, growers, and colleagues at both the Southwest Florida Research and Education Center and the Citrus Research and Education Center in Lake Alfred. The survey is conducted annually in February and March.

RONALD P. MURARO is a Professor of Food and Resource Economics and Extension Farm Management Economist stationed at the Citrus Research and Education Center, Lake Alfred. FRITZ M. ROKA is an Associate Professor in Economics and ROBERT E. ROUSE is an Associate Professor of Horticulture; both are stationed at the Southwest Florida Research and Education Center, Immokalee. 


\section{COSTS AND INPUTS}

Costs for various production inputs are those collected from citrus growers as well as the average of the data obtained from annual custom rate, chemical, and fertilizer surveys. Grower costs are shown in the ADDENDA, Tables 1-A through 7-A. The custom rate charges are shown in Table 8-A and the various chemical and fertilizer costs are shown in Tables 9-A and 10-A in the ADDENDA. Costs of planting and maintaining reset trees through three years of age are shown in Table 13-A. Historic on-tree prices for selected citrus varieties are shown in 16-A. Although brand names are used in many of the tables in the ADDENDA, this does not imply endorsement by the University of Florida. It is merely an attempt to depict typical production practices.

All tables have a column reserved for the individual growers to insert data from a particular grove allowing a comparison of the grower's costs with those presented.

\section{THE GROVE SITUATION}

Production practices for Southwest Florida orange and grapefruit groves are shown in Tables 1 and 2 , respectively, with the times during the year when they are normally performed. There are two benefits to developing such a table for an individual grove. First, it shows what work is needed and when, so that operations can be planned well in advance. Second, it can be helpful if an annual cash flow analysis is developed to plan financing. The individual grower may benefit from developing a plan for a particular grove.

Specific production practices vary from grove to grove making it difficult to define a "typical" grove. Many combinations of practices and various tree variety combinations produce acceptable yields and returns. Although the examples represent Hamlin orange and red seedless grapefruit groves, the cost and return data are designed to be applicable to most grove situations. A grower, realtor, or land appraiser can substitute individual grove costs and expected returns into the budget format and develop a budget for a particular grove. A "your cost" column is appropriately provided for this purpose in subsequent tables.

In the following budget, above average management and cultural practices are assumed. Beyond this general assumption, the following specifics are assumed:

1. A mature (10+ years old), low volume irrigated grove;

2. Varieties are processed-market Hamlin oranges and fresh-market red seedless grapefruit;

3. Annual tree loss is 4.4 trees per acre for oranges and 3.6 trees per acre for grapefruit;

4. Trees are pulled and replaced when production falls below 50 percent of expected yield;

5. Tree density is 145 trees per acre for oranges and 119 trees per acre for grapefruit.

As a result of tree losses and replacement, the tree ages will vary. The orange and grapefruit budgets reflect the following age distributions: 
Table 1.--Schedule of production practices and budget items for a Southwest Florida mature, round orange grove, 2004-05 ${ }^{\text {a }}$

\begin{tabular}{|c|c|c|c|c|c|c|c|c|c|c|c|c|c|}
\hline & & \multicolumn{12}{|c|}{ Month } \\
\hline & & Jan & Feb & Mar & Apr & May & Jun & Jul & Aug & Sep & Oct & Nov & Dec \\
\hline \multicolumn{2}{|l|}{ Total revenue: } & & & $\begin{array}{c}20 \% \\
\text { deposit } \\
\end{array}$ & & $\begin{array}{c}50 \% \\
\text { Partial } \\
\text { payment } \\
\end{array}$ & & & & & & & $\begin{array}{c}\text { Final } \\
\text { payment }\end{array}$ \\
\hline \multirow[t]{2}{*}{ Less: } & Pick \& haul cost & & & & & $\mathrm{X}$ & & & & & & & \\
\hline & DOC advertisement tax & & & & & $\mathrm{X}$ & & & & & & & \\
\hline \multicolumn{14}{|c|}{ Grove expenses: } \\
\hline \multicolumn{2}{|l|}{ Mow } & & $\mathrm{X}$ & & & & $\mathrm{X}$ & $\mathrm{X}$ & $\mathrm{X}$ & & & $\mathrm{X}$ & \\
\hline \multicolumn{2}{|c|}{ Labor, general grove work, pull vines } & $\mathrm{X}$ & & & & & & & & $\mathrm{X}$ & & & \\
\hline \multicolumn{2}{|c|}{ Herbicide ( $1 / 2$ grove acre equivalent) } & & $\mathrm{X}$ & & & & $\mathrm{X}$ & & & $\mathrm{X}$ & & & \\
\hline \multirow[t]{5}{*}{ Spray: } & Dormant & & & & & & & & & & & & \\
\hline & Post bloom/nutritional & & & & $\mathrm{X}$ & & & & & & & & \\
\hline & Supplemental miticide & & & & & & & & & & & & \\
\hline & Summer oil/greasy spot & & & & & & & $\mathrm{X}$ & & & & & \\
\hline & Fall miticide & & & & & & & & & & $\mathrm{X}$ & & \\
\hline \multicolumn{2}{|c|}{ Fertilizer } & \multicolumn{2}{|c|}{$68 \# \mathrm{~N} / \mathrm{A}$} & & & $68 \# \mathrm{~N} / \mathrm{A}$ & & & \multicolumn{2}{|c|}{$68 \# \mathrm{~N} / \mathrm{A}$} & \multicolumn{2}{|c|}{ Dolomite } & \\
\hline \multicolumn{2}{|c|}{ Hedging and topping } & & & Hedge & & & & & & & & & \\
\hline \multicolumn{2}{|c|}{ Brush removal/mow brush } & & \multicolumn{3}{|c|}{ Mow brush } & & & & & & & & \\
\hline \multicolumn{2}{|c|}{ Tree removal } & & $\mathrm{X}$ & & & & & & & & & & \\
\hline \multicolumn{2}{|c|}{ Young tree care } & & & $\mathrm{X}$ & $\mathrm{X}$ & & $\mathrm{X}$ & $\mathrm{X}$ & & $\mathrm{X}$ & & & \\
\hline \multicolumn{2}{|c|}{ Microsprinkler irrigation (times/week) } & 1 & 1 & 2 & 3 & 3 & 3 & 2 & 2 & 2 & 2 & 1 & 1 \\
\hline \multicolumn{2}{|c|}{ Miscellaneous (clean ditches) } & & $\mathrm{X}$ & & & & & & & & & & \\
\hline \multicolumn{2}{|c|}{ Grove taxes including water management } & & & & & & & & & & & $\mathrm{X}$ & \\
\hline \multicolumn{2}{|c|}{ Interest expense } & & & & & & & $\mathrm{X}$ & & & & & \\
\hline \multicolumn{2}{|c|}{ Annual principal payment on mortgage } & & & & & & & $\mathrm{X}$ & & & & & \\
\hline
\end{tabular}

This is a suggested schedule of practices. Actual practices would not necessarily be done on the exact schedule shown here. 


\begin{tabular}{|c|c|c|c|c|c|c|c|c|c|c|c|c|c|}
\hline & & \multicolumn{12}{|c|}{ Month } \\
\hline & & Jan & Feb & Mar & Apr & May & Jun & $\mathrm{Jul}$ & Aug & Sep & Oct & Nov & Dec \\
\hline \multicolumn{2}{|c|}{ Total revenue: } & $\begin{array}{c}20 \% \\
\text { deposit } \\
\end{array}$ & & $\begin{array}{c}\text { Final } \\
\text { payment }\end{array}$ & & & & & & & & & \\
\hline \multirow[t]{2}{*}{ Less: } & Pick \& haul cost & & & $\mathrm{X}$ & & & & & & & & & \\
\hline & DOC advertisement tax & & & $\mathrm{X}$ & & & & & & & & & \\
\hline \multicolumn{14}{|c|}{ Grove expenses: } \\
\hline \multicolumn{14}{|l|}{ Disc } \\
\hline \multicolumn{14}{|l|}{ Chop } \\
\hline Mow & & & $\mathrm{X}$ & & & & $\mathrm{X}$ & $\mathrm{X}$ & $\mathrm{X}$ & & & $\mathrm{X}$ & \\
\hline Labor & general grove work, pull vines & $\mathrm{X}$ & & & & & & & & $\mathrm{X}$ & & & \\
\hline Herbic & de ( $1 / 2$ grove acre equivalent) & & & $\mathrm{X}$ & & & $\mathrm{X}$ & & & & $\mathrm{X}$ & & \\
\hline \multirow[t]{5}{*}{ Spray: } & Dormant & & & & & & & & & & & & \\
\hline & Post bloom/nutritional & & & & $\mathrm{X}$ & & & & & & & & \\
\hline & Supplemental miticide & & & & & $\mathrm{X}$ & & & & & & & \\
\hline & Summer oil/greasy spot & & & & & & $\mathrm{X}$ & & $\mathrm{X}$ & & & & \\
\hline & Fall miticide & & & & & & & & & & $\mathrm{X}$ & & \\
\hline \multicolumn{2}{|c|}{ Fertilizer } & $50 \# \mathrm{~N} / \mathrm{A}$ & & & & $50 \# \mathrm{~N} / \mathrm{A}$ & & & $50 \#$ & $\mathrm{~N} / \mathrm{A}$ & & mite & \\
\hline \multicolumn{2}{|c|}{ Hedging and topping } & & & Hedge & & & & & & & & & \\
\hline \multicolumn{2}{|c|}{ Brush removal/mow brush } & & & Mow brush & & & & & & & & & \\
\hline \multicolumn{2}{|c|}{ Tree removal } & & $\mathrm{X}$ & & & & & & & & & & \\
\hline \multicolumn{2}{|c|}{ Young tree care } & & & $\mathrm{X}$ & $\mathrm{X}$ & & $\mathrm{X}$ & $\mathrm{X}$ & & $\mathrm{X}$ & & & \\
\hline \multicolumn{2}{|c|}{ Microsprinkler irrigation (times/week) } & 1 & 1 & 2 & 3 & 3 & 3 & 2 & 2 & 2 & 2 & 1 & 1 \\
\hline \multicolumn{2}{|c|}{ Miscellaneous (clean ditches) } & & $\mathrm{X}$ & & & & & & & & & & \\
\hline \multicolumn{2}{|c|}{ Grove taxes including water management } & & & & & & & & & & & $\mathrm{X}$ & \\
\hline \multicolumn{2}{|c|}{ Interest expense } & & & & & & & $\mathrm{X}$ & & & & & \\
\hline \multicolumn{2}{|c|}{ Annual principal payment on mortgage } & & & & & & & $\mathrm{X}$ & & & & & \\
\hline
\end{tabular}

${ }^{a}$ This is a suggested schedule of practices. Actual practices would not necessarily be done on the exact schedule shown here. 
$\underline{\text { Hamlin }} \underline{\text { Oranges }}$

\begin{tabular}{rlc}
\multicolumn{2}{l}{ Situation } & Boxes/tree \\
& pulled and reset & 0.0 \\
$3 \%$ & 1 year old & 0.0 \\
$3 \%$ & 2 years old & 0.0 \\
$3 \%$ & 3 years old & 0.7 \\
$3 \%$ & 4 years old & 1.5 \\
$33 \%$ & 5 -15 years old & 4.0 \\
$3 \%$ & producing 50\% of expected yield & 2.2 \\
$49 \%$ & over 15 years old & 4.3
\end{tabular}

and

\section{$\underline{\text { Red }} \underline{\text { Seedless }} \underline{\text { Grapefruit }}$}

\begin{tabular}{rlc}
\multicolumn{2}{c}{ Situation } & Boxes/tree \\
$3 \%$ & pulled and reset & 0.0 \\
$3 \%$ & 1 year old & 0.0 \\
$3 \%$ & 2 years old & 0.0 \\
$3 \%$ & 3 years old & 0.9 \\
$3 \%$ & 4 years old & 1.6 \\
$33 \%$ & 5 -15 years old & 4.0 \\
$3 \%$ & producing 50\% of expected yield & 2.9 \\
$49 \%$ & over 15 years old & 6.5
\end{tabular}

Calculation of normal production per acre for Hamlin oranges and red seedless grapefruit are shown in Tables 3 and 4, respectively.

Table 3.--Calculation of normal production per tree and per acre for Hamlin oranges, 2004-05

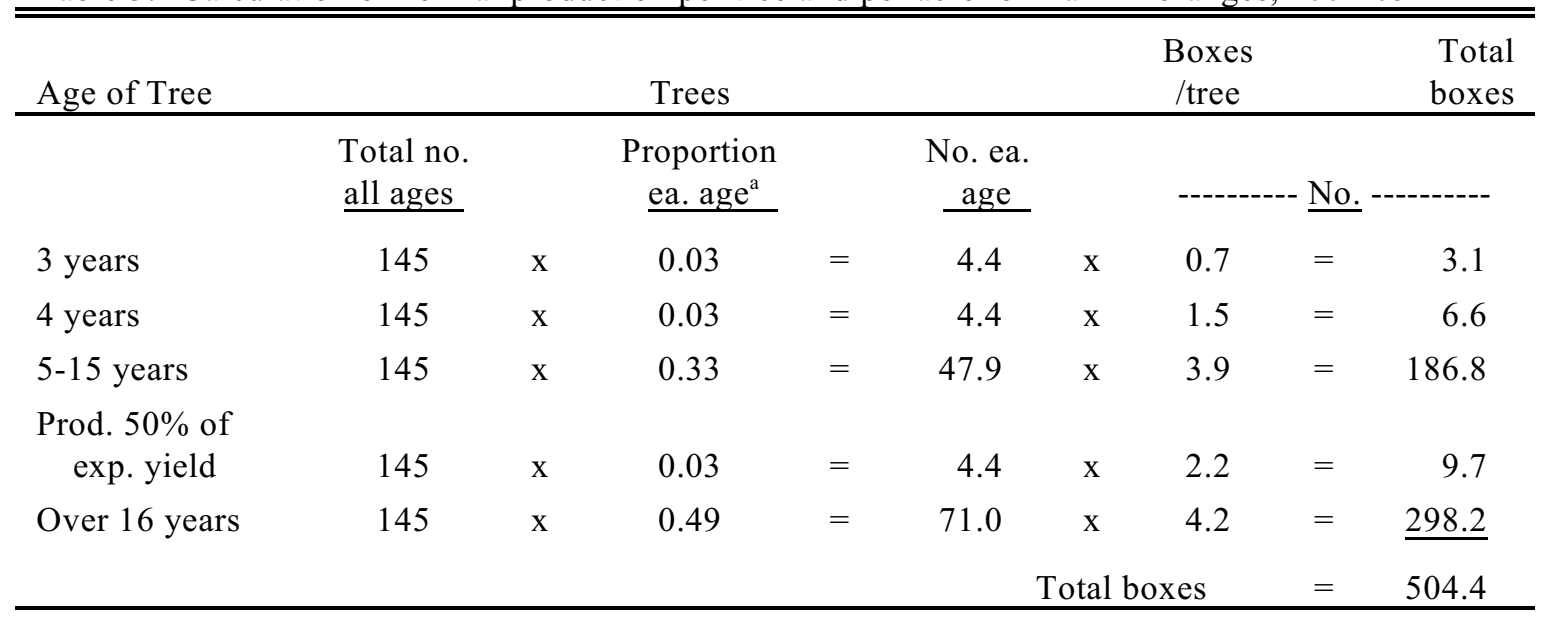

a Proportion adds up to 91 percent as 9 percent of the trees were non-bearing (pulled and reset, 1 and 2 year old trees). 
Table 4.--Calculation of normal production per tree and per acre for red seedless grapefruit, 2004-05

\begin{tabular}{|c|c|c|c|c|c|c|c|c|c|}
\hline Age of Tree & & & Trees & & & & $\begin{array}{r}\text { Boxes } \\
/ \text { tree }\end{array}$ & & $\begin{array}{r}\text { Total } \\
\text { boxes }\end{array}$ \\
\hline & $\begin{array}{l}\text { Total no. } \\
\text { all ages }\end{array}$ & & $\begin{array}{c}\text { Proportion } \\
\text { ea. age }^{\mathrm{a}} \\
\end{array}$ & & $\begin{array}{c}\text { No. ea. } \\
\text { age }\end{array}$ & & ---- & No & ------- \\
\hline 3 years & 119 & $\mathrm{x}$ & 0.03 & $=$ & 3.6 & $\mathrm{x}$ & 0.9 & $=$ & 3.2 \\
\hline 4 years & 119 & $\mathrm{x}$ & 0.03 & $=$ & 3.6 & $\mathrm{x}$ & 1.6 & $=$ & 5.8 \\
\hline $5-15$ years & 119 & $\mathrm{x}$ & 0.33 & $=$ & 39.3 & $\mathrm{x}$ & 4.0 & $=$ & 157.2 \\
\hline $\begin{array}{l}\text { Prod. } 50 \% \text { of } \\
\text { exp. yield }\end{array}$ & 119 & $\mathrm{x}$ & 0.03 & $=$ & 3.6 & $\mathrm{x}$ & 2.9 & $=$ & 10.4 \\
\hline Over 16 years & 119 & $\mathrm{x}$ & 0.49 & $=$ & 58.3 & $\mathrm{x}$ & 6.5 & $=$ & $\underline{379.0}$ \\
\hline & & & & & \multicolumn{3}{|c|}{ Total boxes } & $=$ & 555.6 \\
\hline
\end{tabular}

${ }^{\text {a }}$ Proportion adds up to 91 percent as 9 percent of the trees were non-bearing (pulled and reset, 1 and 2 year old trees; see page 5 ).

\section{BUDGET COSTS AND RETURNS}

The estimated budget costs and returns for the two Southwest Florida grove situations are shown in Tables 5 and 7 . The budgeted costs represent one possible citrus production program and were selected from the costs shown in the ADDENDA tables. The costs presented in the budgets represent an owner-managed citrus operation. The gross revenue estimates are based on the projected yields in Tables 3 and 4 and estimated preliminary on-tree prices for the 2004-05 season. Alternative cost scenarios, grove reset costs, harvesting and packing charges can be found in Tables 11-A through 15-A in the ADDENDA. Also, historical on-tree prices for selected Florida citrus varieties are shown in Table 16-A of the ADDENDA.

As shown in Table 5, the total revenue for processed-market Hamlin oranges is estimated to be $\$ 1,149.12$ per acre. Total specified costs are $\$ 881.03$ and are comprised of grove care costs of $\$ 833.03$, plus management cost of $\$ 48.00$. Return to land and trees which represents net return above variable costs was $\$ 268.09$ per acre. At 350 and 550 boxes per acre, respectively, the break-even price required to cover grove care costs for Hamlin oranges range from $\$ 2.38$ to $\$ 1.52$ per box on-tree and $\$ 0.82$ to $\$ 0.68$ per pounds solids delivered-in.

In Table 7, total revenue for fresh market red seedless grapefruit is estimated to be $\$ 7,919.85$ per acre. Total specified costs are $\$ 997.74$, being comprised of grove care costs of $\$ 949.74$, plus management cost of $\$ 48.00$. Return to land, trees, and ownership or net return above variable costs is $\$ 6,922.11$. At 350 and 550 boxes per acre, respectively, the break-even price required to cover grove care costs for seedless grapefruit range from $\$ 2.72$ to $\$ 1.73$ per box on-tree and $\$ 1.39$ to $\$ 1.18$ per pound solids delivered-in for eliminations. 
Ad valorem taxes, and overhead and administrative costs ( such as water drainage district taxes, crop insurance, and other grower assessments) can add up to 12 percent of the total grove care costs. These costs vary from grove to grove depending on age, location, variety of fruit, etc. and should be considered in arriving at a net return to land, trees, and ownership (total return minus total costs). Harvest costs (pick, roadside, and hauling costs) also add to the total fruit cost delivered to either a processing plant or fresh fruit packinghouse. Also, average annual debt payment (principal and interest) may be as high as $\$ 435$ per acre $(\$ 3,700$ average debt per acre @10 percent interest amortized over 20 years $)$ which would reduce total available cash for grove expansion or other investment.

An estimated "delivered-in" costs are shown for processed oranges in Table 6 and fresh packed red grapefruit in Table 8. "Delivered-in" costs include grove care costs (Tables 5 and 7) plus harvesting, regulatory, and grower assessment costs. The "delivered-in" costs are presented as a cost per acre, per box, and per pound solids or per carton. Three possible budget cost scenarios are presented (Refer to Tables 11-A and 12-A): 1) Low Cost Processed Cultural Program; 2) Reduced Cost Cultural Program; and 3) Typical/Historical Cultural Program. Scenarios 1 and 2 represent costs of two possible cultural programs directed toward reducing the expenditures for fruit grown primarily for the fresh/processed market. The third scenario represents typical costs of grove practices which have been performed for citrus grown for the fresh fruit market in the case of grapefruit and the processed market in the case or oranges. Modified herbicide and/or spray and fertilizer programs account for the reduced costs. NOTE: $\underline{\text { Before modifying a grove management program to reduce costs, an evaluation of the market program }}$ (processed or fresh), yield, and specific cultural problems (nutrition, disease, etc.) for the specific grove site should be made. Also, in Table 9, the total estimated F.O.B. cost for fresh packed Red grapefruit is shown. The F.O.B. costs are presented for "fresh fruit packout percentage rates" ranging from 50 percent to 100 percent.

\section{HISTORICAL COST TRENDS}

Annual budgets of costs and returns for mature, processed Hamlin oranges and mature fresh-market seedless grapefruit in the Southwest Florida area have been developed and published in each of the past five years. Estimated cost and return histories for 2000-01 through 2003-04 along with 2004-05, and a five-year average are presented in Tables 10 and 12. To allow comparisons in current values, these same costs and returns, adjusted to 2005 dollars, are presented in Tables 11 and 13. 


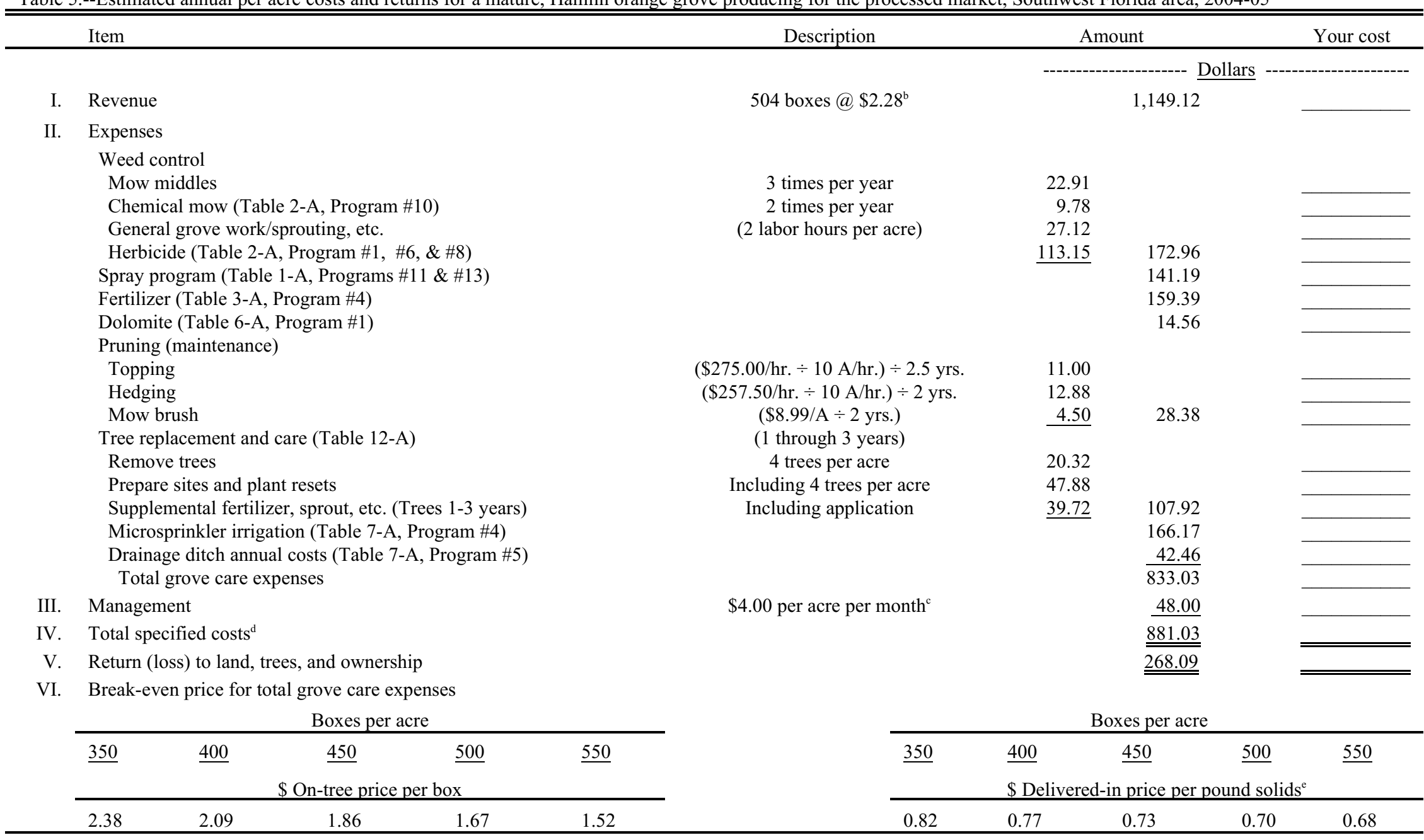

${ }^{a}$ Although the estimated annual per acre grove costs shown in Tables 5 and 6 are representative for a mature Southwest Florida Hamlin orange and red seedless grapefruit grove, respectively, the grove care costs for a specific grove site may differ depending upon the grove practices performed; e.g., a Temik application would add $\$ 116.94$ per acre; extensive tree loss due to blight or tristeza could at least double, if not increase more, the tree replacement and care costs; etc. Also, truck watering of resets could add another $\$ 8.90$ per acre (average 5 waterings).

${ }^{b} \mathrm{On}$-tree price per box is preliminary; assumes price for processed oranges only.

'Other methods to estimate a management cost--e.g., $5 \%$ of gross sales or $10 \%$ of total grove care costs--are used in the industry. Other selected methods will give a different return to land and trees than reported here.

dOther cost items which are not included in the budget are ad valorem taxes and interest on grove investment. In addition to these cost items, overhead and administrative costs, such as water drainage/district taxes,

crop insurance, and other grower assessments, can add up to 12 percent to the total grove care costs. These costs vary from grove to grove depending on age, location, and time of purchase or grove establishment

${ }^{\mathrm{C} A s s u m e s} 6.0$ pounds solids per box and $\$ 2.357$ pick and haul cost per box (including canker decontamination costs) and Department of Citrus advertising assessment of $\$ 0.165$ per box. 
Table 6.--Estimated total delivered-in cost for Southwest Florida Hamlin oranges grown for the processed market under three cultural cost programs, 2004-05

\begin{tabular}{|c|c|c|c|c|c|c|c|c|c|}
\hline \multirow[t]{2}{*}{$\begin{array}{l}\text { Represents a mature }(10+\text { years old }) \\
\text { Southwest Florida Orange Grove }\end{array}$} & \multicolumn{3}{|c|}{$\begin{array}{c}\text { Processed Hamlin Oranges } \\
\text { Low Cost Cultural Program } \\
\text { One-Year Alternative }\end{array}$} & \multicolumn{3}{|c|}{$\begin{array}{c}\text { Processed Hamlin Oranges } \\
\text { Low Cost } \\
\text { Cultural Program }\end{array}$} & \multicolumn{3}{|c|}{$\begin{array}{c}\text { Fresh/Processed Hamlin Oranges } \\
\text { Historical Cost } \\
\text { Cultural Program }\end{array}$} \\
\hline & $\$ /$ Acre & $\$ /$ Box & \$/P.S. & $\$ /$ Acre & $\$ /$ Box & \$/P.S. & $\$ /$ Acre & $\$ /$ Box & \$/P.S. \\
\hline Total Production/Cultural Costs & $\$ 735.99$ & $\$ 1.460$ & $\$ 0.2434$ & $\$ 833.03$ & $\$ 1.653$ & $\$ 0.2755$ & $\$ 954.72$ & $\$ 1.894$ & $\$ 0.3157$ \\
\hline Interest on Operating (Cultural) Costs & 20.24 & 0.040 & 0.0067 & 41.65 & 0.083 & 0.0138 & 47.74 & 0.095 & 0.0158 \\
\hline Management Costs & 48.00 & 0.095 & 0.0159 & 48.00 & 0.095 & 0.0159 & 48.00 & 0.095 & 0.0159 \\
\hline \multicolumn{10}{|l|}{ Taxes/Regulatory Costs: } \\
\hline $\begin{array}{l}\text { Property Tax and Water Management } \\
\text { District Tax }\end{array}$ & 64.05 & 0.127 & 0.0212 & 61.00 & 0.121 & 0.0202 & 61.00 & 0.121 & 0.0202 \\
\hline Canker Decontamination Costs & 6.18 & $\underline{0.012}$ & $\underline{0.0020}$ & 4.54 & $\underline{0.009}$ & $\underline{0.0015}$ & 4.54 & $\underline{0.009}$ & $\underline{0.0015}$ \\
\hline Total Direct Grower Costs & $\$ 874.46$ & $\$ 1.735$ & $\$ 0.2892$ & $\$ 988.22$ & $\$ 1.961$ & $\$ 0.3268$ & $\$ 1,116.00$ & $\$ 2.214$ & $\$ 0.3690$ \\
\hline Interest on Avg Capital Investment Costs & 321.22 & $\underline{0.637}$ & $\underline{\$ 0.1062}$ & 321.22 & $\underline{0.637}$ & $\underline{0.1062}$ & 321.22 & $\underline{0.637}$ & $\underline{0.1062}$ \\
\hline Total Grower Costs & $\$ 1,195.67$ & $\$ 2.372$ & $\$ 0.3954$ & $\$ 1,309.44$ & $\$ 2.598$ & $\$ 0.4330$ & $\$ 1,437.21$ & $\$ 2.852$ & $\$ 0.4753$ \\
\hline \multicolumn{10}{|l|}{ Harvesting and Assessment Costs: } \\
\hline $\begin{array}{l}\text { Pick/Spot Pick, Roadside \& Haul and } \\
\text { Canker Decontamination Costs }\end{array}$ & $1,187.93$ & 2.357 & 0.3928 & $1,187.93$ & 2.357 & 0.3928 & $1,187.93$ & 2.357 & 0.3928 \\
\hline DOC Assessment & 83.16 & $\underline{0.165}$ & $\underline{0.0275}$ & 83.16 & $\underline{0.165}$ & $\underline{0.0275}$ & 83.16 & $\underline{0.165}$ & $\underline{0.0275}$ \\
\hline Total Harvesting \& Assessment Costs & $1,271.09$ & 2.522 & 0.4203 & $1,271.09$ & 2.522 & 0.4203 & $1,271.09$ & 2.522 & 0.4203 \\
\hline Total Delivered-In Cost & $\$ \underline{\underline{2,466.76}}$ & $\$ \underline{\underline{4.894}}$ & $\$ \underline{\underline{0.8157}}$ & $\$ \underline{\underline{2,580.52}}$ & $\$ \underline{\underline{5.120}}$ & $\$ \underline{\underline{0.8533}}$ & $\$ \underline{\underline{2,708.30}}$ & $\$ \underline{\underline{5.374}}$ & $\$ \underline{\underline{0.8956}}$ \\
\hline $\begin{array}{l}\text { P.S. = Pound Solids } \\
\text { Yield: } 504 \text { boxes/acre @ } 6.0 \text { P.S. per box } \\
145 \text { trees per acre }\end{array}$ & \multicolumn{3}{|c|}{$\begin{array}{l}\text { Refer to cultural program shown } \\
\text { in Table 11-A. } \\
\text { Only summer oil sprays with oil, } \\
\text { copper, and Agri-mek \& } \\
\text { nutritionals. }\end{array}$} & \multicolumn{3}{|c|}{$\begin{array}{c}\text { Refer to cultural program shown } \\
\text { in Table } 5 .\end{array}$} & \multicolumn{3}{|c|}{$\begin{array}{l}\text { Refer to cultural program shown } \\
\text { in Table 11-A. } \\
\text { A Fall Miticide Spray added to the } \\
\text { cultural program shown in Table } 5 .\end{array}$} \\
\hline
\end{tabular}




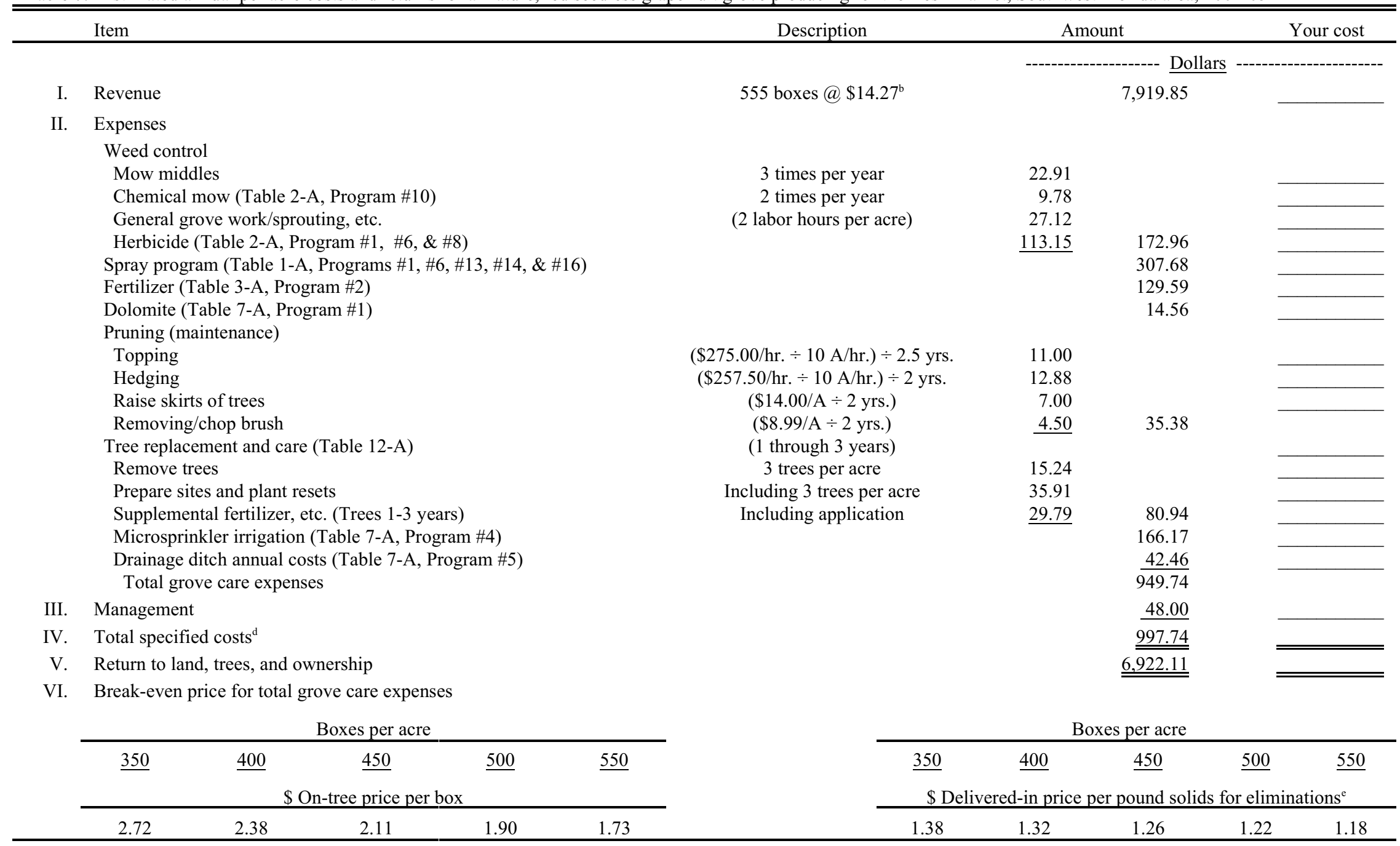

${ }^{a}$ Although the estimated annual per acre grove costs shown in Tables 5 and 6 are representative for a mature Southwest Florida Hamlin orange and red seedless grapefruit grove, respectively, the grove care costs for a specific grove site may differ depending upon the grove practices performed; e. $g$., a Temik application would add \$116.94 per acre; extensive tree loss due to blight or tristeza could at least double, if not increase more, the tree replacement and care costs; etc. Also, truck watering of resets could add another $\$ 8.90$ per acre (average 5 waterings).

${ }^{b} \mathrm{On}$-tree price per box is preliminary; assumes average of all methods of sale (fresh and processed).

${ }^{\mathrm{c}}$ Other methods to estimate a management cost--e.g., $5 \%$ of gross sales or $10 \%$ of total grove care costs--are used in the industry. Other selected methods will give a different return to land and trees than reported here.

${ }^{d}$ Other cost items which are not included in the budget are ad valorem taxes and interest on grove investment. In addition to these cost items, overhead and administrative costs, such as water drainage/district taxes, crop insurance, and other grower assessments, can add up to 12 percent to the total grove care costs. These costs vary from grove to grove depending on age, location, and tim of purchase or grove establishment.

${ }^{\circ}$ Assumes 4.7 pounds solids per box; $\$ 2.809$ pick and haul cost per box (includes spot picking, fruit drenching and canker decontamination costs and D.O.C. $\$ 0.25$ advertising tax), $\$ 0.55$ per box handling through packinghouse, and $\$ 0.45$ per box delivery to processing plant. 
Table 8.--Estimated total delivered-in cost for Southwest Florida Red Grapefruit grown for the fresh/processed market under three cultural cost programs,

2004-05

\begin{tabular}{|c|c|c|c|c|c|c|c|c|c|}
\hline \multirow[t]{2}{*}{$\begin{array}{l}\text { Represents a mature }(10+\text { years old }) \\
\text { Southwest Florida Red Grapefruit Grove }\end{array}$} & \multicolumn{3}{|c|}{$\begin{array}{c}\text { Processed Red Grapefruit } \\
\text { Low Cost Cultural Program } \\
\text { One-Year Alternative }\end{array}$} & \multicolumn{3}{|c|}{$\begin{array}{l}\text { Fresh Packed Red Grapefruit } \\
\text { Reduced Cost } \\
\text { Cultural Program }\end{array}$} & \multicolumn{3}{|c|}{$\begin{array}{c}\text { Fresh Packed Red Grapefruit } \\
\text { Typical/Historical } \\
\text { Cultural Program }\end{array}$} \\
\hline & $\$ /$ Acre & \$/Box & \$/P.S. & $\$ /$ Acre & $\$ /$ Box & \$/Carton & $\$ /$ Acre & $\$ /$ Box & $\$ /$ Carton \\
\hline Total Production/Cultural Costs & $\$ 701.81$ & $\$ 1.265$ & $\$ 0.2690$ & $\$ 904.94$ & $\$ 1.631$ & $\$ 1.0144$ & $\$ 949.74$ & $\$ 1.711$ & $\$ 1.0144$ \\
\hline Interest on Operating (Cultural) Costs & 19.30 & 0.035 & 0.0074 & 24.89 & 0.045 & 0.0224 & 26.12 & 0.047 & 0.0235 \\
\hline Management Costs & 48.00 & 0.086 & 0.0184 & 48.00 & 0.086 & 0.0432 & 48.00 & 0.086 & 0.0432 \\
\hline $\begin{array}{l}\text { Taxes/Regulatory Costs: } \\
\text { Property Tax and Water Management } \\
\text { District Tax } \\
\text { Fly Protocol Cost } \\
\text { Canker Decontamination Costs }\end{array}$ & $\begin{array}{c}51.24 \\
- \\
6.18 \\
\end{array}$ & $\begin{array}{c}0.092 \\
- \\
0.011 \\
\end{array}$ & $\begin{array}{c}0.0196 \\
- \\
0.0024 \\
\end{array}$ & $\begin{array}{r}51.24 \\
54.73 \\
6.18 \\
\end{array}$ & $\begin{array}{l}0.092 \\
0.099 \\
0.011 \\
\end{array}$ & $\begin{array}{l}0.0478 \\
0.0477 \\
0.0016 \\
\end{array}$ & $\begin{array}{r}51.24 \\
54.73 \\
6.18 \\
\end{array}$ & $\begin{array}{l}0.092 \\
0.099 \\
0.011 \\
\end{array}$ & $\begin{array}{l}0.0478 \\
0.0477 \\
0.0016 \\
\end{array}$ \\
\hline Total Taxes/Regulatory Costs & 57.42 & $\underline{0.103}$ & $\underline{0.0220}$ & 112.15 & $\underline{0.202}$ & $\underline{0.0971}$ & 112.15 & $\underline{0.202}$ & $\underline{0.0971}$ \\
\hline Total Direct Grower Costs & $\$ 826.53$ & $\$ 1.489$ & $\$ 0.3169$ & $\$ 1,089.98$ & $\$ 1.964$ & $\$ 1.1772$ & $\$ 1,136.01$ & $\$ 2.047$ & $\$ 1.1783$ \\
\hline Interest on Average Capital Investment Costs & 321.22 & $\underline{0.579}$ & $\underline{0.1231}$ & 321.22 & $\underline{0.579}$ & $\underline{0.2894}$ & 321.22 & $\underline{0.579}$ & $\underline{0.2894}$ \\
\hline Total Grower Costs & $\$ 1,147.74$ & $\$ 2.068$ & $\$ 0.4400$ & $\$ 1,411.19$ & $\$ 2.543$ & $\$ 1.4666$ & $\$ 1,457.22$ & $\$ 2.626$ & $\$ 1.4677$ \\
\hline $\begin{array}{l}\text { Harvesting and Assessment Costs: } \\
\text { Pick/Spot Pick, Roadside \& Haul and } \\
\text { Canker Decontamination } \\
\text { Fruit Drenching (Fresh) } \\
\text { DOC Assessment }\end{array}$ & $\begin{array}{c}1,207.13 \\
- \\
133.20 \\
\end{array}$ & $\begin{array}{c}2.175 \\
- \\
0.240 \\
\end{array}$ & $\begin{array}{c}0.4628 \\
- \\
0.0511 \\
\end{array}$ & $\begin{array}{r}1,317.57 \\
102.68 \\
138.75 \\
\end{array}$ & $\begin{array}{l}2.374 \\
0.185 \\
0.250 \\
\end{array}$ & $\begin{array}{l}1.1870 \\
0.0925 \\
0.1250 \\
\end{array}$ & $\begin{array}{r}1,317.57 \\
102.68 \\
138.75 \\
\end{array}$ & $\begin{array}{l}2.374 \\
0.185 \\
0.250 \\
\end{array}$ & $\begin{array}{l}1.1870 \\
0.0925 \\
0.1250 \\
\end{array}$ \\
\hline Total Harvesting and Assessment Costs & $1,340.33$ & 2.415 & 0.5138 & $1,559.00$ & 2.809 & 1.4045 & $1,559.00$ & 2.809 & 1.4045 \\
\hline Total Delivered-In Cost & $\$ 2,488.07$ & $\$ \underline{\underline{4.483}}$ & $\$ \underline{\underline{0.9538}}$ & $\$ 2,970.19$ & $\$ 5.352$ & $\$ 2.8711$ & $\$ \underline{\underline{3,016.22}}$ & $\$ \underline{\underline{\underline{5.435}}}$ & $\$ 2.8722$ \\
\hline $\begin{array}{l}\text { Two cartons per box } \\
\text { P.S.= Pound Solids } \\
\text { Yield: } 555 \text { boxes/acre @ } 4.7 \text { P.S. per box } \\
119 \text { trees per acre }\end{array}$ & $\begin{array}{r}\text { Two summ } \\
\text { copper }\end{array}$ & $\begin{array}{l}\text { ultural } \mathrm{p} \\
\text { n Table } 1\end{array}$ & $\begin{array}{l}\text { with oil, } \\
\text { nek. }\end{array}$ & Refer to cu & $\begin{array}{l}\text { ural prog } \\
\text { Table } 7 \text {. }\end{array}$ & am shown & $\begin{array}{r}\text { Refer to } \mathrm{c} \\
\text { is }\end{array}$ & $\begin{array}{l}\text { aral progr } \\
\text { able } 12-A\end{array}$ & im shown \\
\hline
\end{tabular}


Table 9.--Estimated F.O.B. cost for fresh market Southwest Florida Red grapefruit, 2004-05

\begin{tabular}{|c|c|c|c|c|c|c|c|c|c|}
\hline & \multicolumn{3}{|c|}{$\begin{array}{lc}\text { Percent Packout } & 50.00 \% \\
\text { Box Yield Per Acre } & 555\end{array}$} & \multicolumn{3}{|c|}{$\begin{array}{lc}\text { Percent Packout } & 60.00 \% \\
\text { Box Yield Per Acre } & 555\end{array}$} & \multicolumn{3}{|c|}{$\begin{array}{lc}\text { Percent Packout } & 70.00 \% \\
\text { Box Yield Per Acre } & 555\end{array}$} \\
\hline & Per Acre & Per Box & Per Carton & Per Acre & Per Box & Per Carton & Per Acre & Per Box & Per Carton \\
\hline $\begin{array}{l}\text { Total Production/ } \\
\text { Cultural Costs }\end{array}$ & $\$ 949.74$ & $\$ 4.268$ & $\$ 2.1342$ & $\$ 949.74$ & $\$ 3.557$ & $\$ 1.7785$ & $\$ 949.74$ & $\$ 3.049$ & $\$ 1.5245$ \\
\hline $\begin{array}{l}\text { Interest on Operating } \\
\text { (Cultural Costs) }\end{array}$ & 26.12 & 0.117 & 0.0587 & 26.12 & 0.098 & 0.0489 & 26.12 & 0.084 & 0.0419 \\
\hline Management & 48.00 & 0.216 & 0.1079 & 48.00 & 0.180 & 0.0899 & 48.00 & 0.154 & 0.0770 \\
\hline Taxes/Regulatory & 112.15 & 0.504 & 0.2520 & 112.15 & 0.420 & 0.2100 & 112.15 & 0.360 & 0.1800 \\
\hline $\begin{array}{l}\text { Interest on Average } \\
\text { Capital Investment }\end{array}$ & 321.22 & 1.444 & 0.7218 & 321.22 & 1.203 & 0.6015 & 321.22 & 1.031 & 0.5156 \\
\hline $\begin{array}{l}\text { Harvesting (Pick, Haul, Etc.) } \\
\text { and DOC Assessment }\end{array}$ & $\underline{1,559.00}$ & 7.007 & $\underline{3.5034}$ & $\underline{1,559.00}$ & $\underline{5.839}$ & $\underline{2.9195}$ & $\underline{1,559.00}$ & $\underline{5.005}$ & $\underline{2.5024}$ \\
\hline Total Delivered-In Cost & $\$ 3,016.22$ & $\$ 13.556$ & $\$ 6.7780$ & $\$ 3,016.22$ & $\$ 11.297$ & $\$ 5.6483$ & $\$ 3,016.22$ & $\$ 9.683$ & $\$ 4.8414$ \\
\hline Packing \& Selling & $2,047.95$ & 7.380 & 3.6900 & $2,457.54$ & 7.380 & 3.6900 & $2,867.13$ & 7.380 & 3.6900 \\
\hline $\begin{array}{l}\text { Net Costs of Fresh } \\
\text { Eliminations }{ }^{\mathrm{a}}\end{array}$ & $-2,096.24$ & $-\underline{9.421}$ & $-\underline{-4.7106}$ & $-1,676.99$ & $\underline{-6.281}$ & $-\underline{3.1404}$ & $-1,257.74$ & $\underline{-4.038}$ & -2.0188 \\
\hline \multirow[t]{3}{*}{ Total F.O.B. Costs } & $\$ \underline{\underline{2,967.93}}$ & $\$ 11.515$ & $\$ 5.7574$ & $\$ \underline{\underline{3,796.77}}$ & $\$ 12.396$ & $\$ \underline{\underline{6.1979}}$ & $\$ \underline{\underline{4,625.61}}$ & $\$ 13.025$ & $\$ 6.5126$ \\
\hline & \multicolumn{3}{|c|}{$\begin{array}{lc}\text { Percent Packout } & 80.00 \% \\
\text { Box Yield Per Acre } & 555\end{array}$} & \multicolumn{3}{|c|}{$\begin{array}{lc}\text { Percent Packout } & 90.00 \% \\
\text { Box Yield Per Acre } & 555\end{array}$} & \multicolumn{3}{|c|}{$\begin{array}{lc}\text { Percent Packout } & 100.00 \% \\
\text { Box Yield Per Acre } & 555\end{array}$} \\
\hline & Per Acre & Per Box & Per Carton & Per Acre & Per Box & Per Carton & Per Acre & Per Box & Per Carton \\
\hline $\begin{array}{l}\text { Total Production/ } \\
\text { Cultural Costs }\end{array}$ & $\$ 949.74$ & $\$ 2.668$ & $\$ 1.3339$ & $\$ 949.74$ & $\$ 2.371$ & $\$ 1.1857$ & $\$ 949.74$ & $\$ 2.134$ & $\$ 1.0671$ \\
\hline $\begin{array}{l}\text { Interest on Operating } \\
\text { (Cultural) Costs }\end{array}$ & 26.12 & 0.073 & 0.0367 & 26.12 & 0.065 & 0.0326 & 26.12 & 0.059 & 0.0293 \\
\hline Management & 48.00 & 0.135 & 0.0674 & 48.00 & 0.120 & 0.0599 & 48.00 & 0.108 & 0.0539 \\
\hline Taxes/Regulatory & 112.15 & 0.315 & 0.1575 & 112.15 & 0.280 & 0.1400 & 112.15 & 0.252 & 0.1260 \\
\hline $\begin{array}{l}\text { Interest on Average } \\
\text { Capital Investment }\end{array}$ & 321.22 & 0.902 & 0.4511 & 321.22 & 0.802 & 0.4010 & 321.22 & 0.722 & 0.3609 \\
\hline $\begin{array}{l}\text { Harvesting (Pick, Haul, Etc.) } \\
\text { and DOC Assessment }\end{array}$ & $\underline{1,559.00}$ & 4.379 & $\underline{2.1896}$ & $\underline{1,559.00}$ & 3.893 & $\underline{1.9463}$ & $\underline{1,559.00}$ & 3.503 & $\underline{1.7517}$ \\
\hline Total Delivered-In Cost & $\$ 3,016.22$ & $\$ 8.473$ & $\$ 4.2363$ & $\$ 3,016.22$ & $\$ 7.531$ & $\$ 3.7656$ & $\$ 3,016.22$ & $\$ 6.778$ & $\$ 3.3890$ \\
\hline Packing \& Selling & $3,276.72$ & 7.380 & 3.6900 & $3,686.31$ & 7.380 & 3.6900 & $4,095.90$ & 7.380 & 3.6900 \\
\hline $\begin{array}{l}\text { Net Costs of Fresh } \\
\text { Eliminations }\end{array}$ & -838.49 & $-\underline{2.355}$ & $-\underline{-1.1777}$ & -419.25 & -1.047 & $-\underline{0.5234}$ & 0.00 & 0.000 & $\underline{0.0000}$ \\
\hline Total F.O.B. Costs & $\$ 5,454.44$ & $\$ 13.497$ & $\$ \underline{6.7486}$ & $\$ 6,283.28$ & $\$ 13.864$ & $\$ \underline{6.9322}$ & $\$ 7,112.12$ & $\$ 14.158$ & $\$ 7.0790$ \\
\hline
\end{tabular}

a"Net Eliminations Cost" equals the average yield of 4.70 pound solids per box times $\$ 1.82$ per pound solids less packinghouse elimination charge and cannery hauling charge of $\$ 1.00$ per box. 
Table 10.--Estimated annual per acre costs and returns and 5-year average costs and returns for a mature, Hamlin orange grove producing citrus for processed market in the Southwest Florida area, 2000-01-2004-05

\begin{tabular}{|c|c|c|c|c|c|c|}
\hline Year & $\begin{array}{l}\text { On-tree } \\
\text { price/box }{ }^{\mathrm{a}}\end{array}$ & Yield & $\begin{array}{l}\text { Gross } \\
\text { revenue }\end{array}$ & $\begin{array}{c}\text { Total grove } \\
\text { care expenses }\end{array}$ & $\begin{array}{c}\text { Total specified } \\
\text { costs }^{\mathrm{c}}\end{array}$ & $\begin{array}{l}\text { Net return to land, } \\
\text { trees, and ownership }\end{array}$ \\
\hline & & & 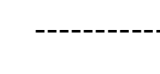 & 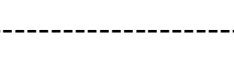 & ollars ------- & - \\
\hline 2000-01 & $\$ 2.57$ & 504 & $1,295.28$ & 769.04 & 817.04 & 478.14 \\
\hline 2001-02 & $\$ 2.79$ & 504 & $1,406.16$ & 767.23 & 815.23 & 590.93 \\
\hline $2002-03$ & $\$ 2.65$ & 504 & $1,335.60$ & 771.03 & 819.03 & 516.57 \\
\hline 2003-04 & $\$ 2.11$ & 516 & $1,088.76$ & 768.21 & 816.21 & 272.55 \\
\hline 2004-05 & $\$ 2.28^{\mathrm{b}}$ & 504 & $1,149.12$ & 833.03 & 881.03 & 268.09 \\
\hline 5-yr. avg. & $\$ 2.48$ & 506 & $1,254.88$ & 781.71 & 830.71 & 424.17 \\
\hline
\end{tabular}

${ }^{a}$ On-tree prices for processed oranges only as reported by the Florida Agricultural Statistics Service.

${ }^{\mathrm{b}}$ Preliminary estimate by FASS for 2004-05 season.

${ }^{\mathrm{c}} \mathrm{A}$ management cost of $\$ 4.00$ per acre per month is included. Fixed costs such as taxes, debt service, and crop insurance are not included. 
Table 11.--Estimated annual per acre costs and returns and 5-year average costs and returns (adjusted to 2005 dollars) for a mature, Hamlin orange grove producing citrus for processed market in the Southwest Florida area, 2000-01-2004-05

\begin{tabular}{|c|c|c|c|c|c|c|}
\hline Year & $\begin{array}{c}\text { Inflation } \\
\text { factor index }^{\mathrm{a}}\end{array}$ & $\begin{array}{c}\text { Adjusted } \\
\text { on-tree } \\
\text { price/box }\end{array}$ & Yield & $\begin{array}{c}\text { Gross } \\
\text { revenue }\end{array}$ & $\begin{array}{c}\text { Total specified } \\
\text { costs }^{\mathrm{b}}\end{array}$ & $\begin{array}{l}\text { Net return to land, } \\
\text { trees, and ownership }\end{array}$ \\
\hline & & & & --------- & Dollars & 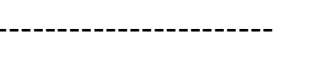 \\
\hline $2000-01$ & 117.9 & $\$ 3.03$ & 504 & $1,527.12$ & 963.29 & 563.83 \\
\hline $2001-02$ & 120.7 & $\$ 3.37$ & 504 & $1,698.48$ & 983.99 & 714.49 \\
\hline $2002-03$ & 114.6 & $\$ 3.04$ & 504 & $1,532.16$ & 938.61 & 593.55 \\
\hline 2003-04 & 107.9 & $\$ 2.28$ & 516 & $1,176.48$ & 880.69 & 295.29 \\
\hline 2004-05 & 100.0 & $\$ 2.28$ & 504 & $1,149.12$ & 881.03 & 268.09 \\
\hline 5-yr. avg. & - & $\$ 2.80$ & 506 & $1,416.80$ & 929.53 & 487.27 \\
\hline
\end{tabular}

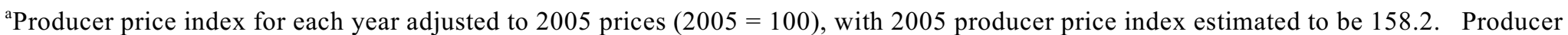
price index for other years are: $2001=134.2 ; 2002=131.1 ; 2003=138.1$; and $2004=146.7$.

${ }^{\mathrm{b}} \mathrm{A}$ management cost of $\$ 4.00$ per acre per month is included. Fixed costs such as taxes, debt service, and crop insurance are not included. (Refer to Table 10.) 
Table 12.--Estimated annual per acre costs and returns and 5-year average costs and returns for a mature, red seedless grapefruit grove producing citrus for fresh market packing in the Southwest Florida area, 2000-01-2004-05

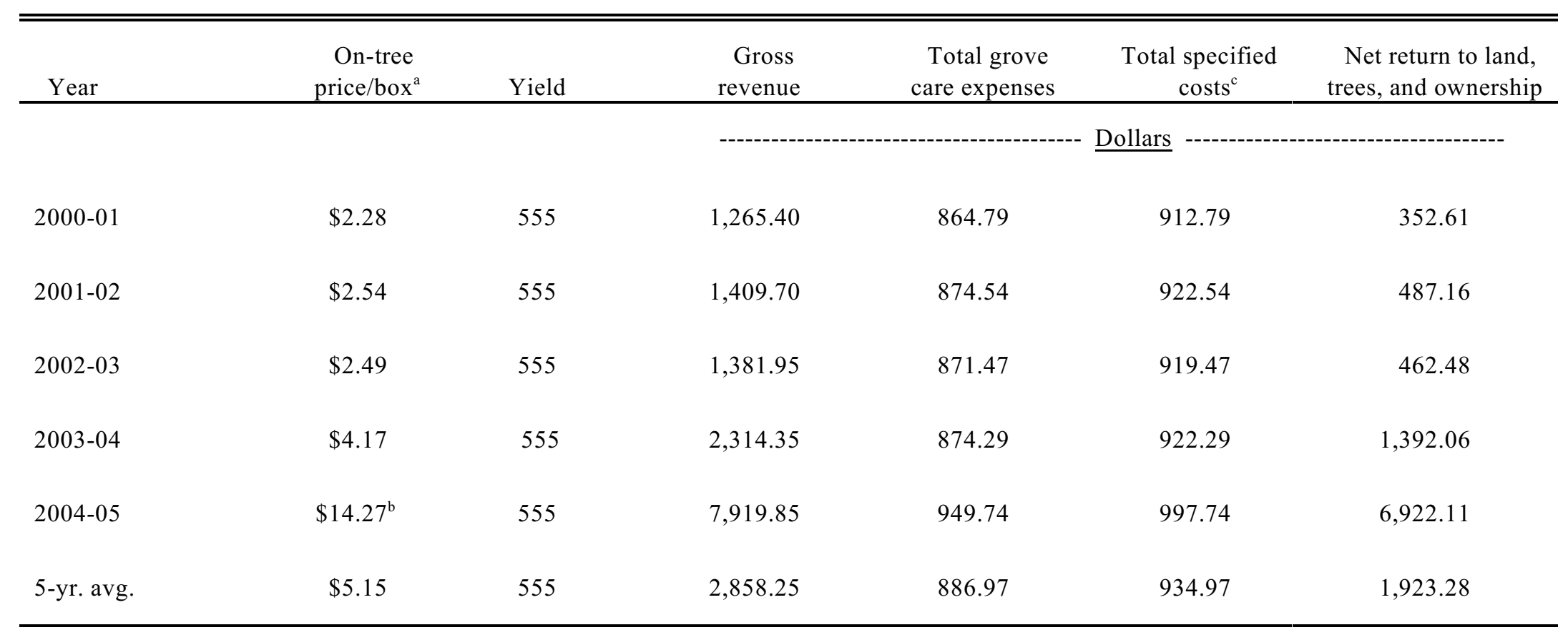

${ }^{\mathrm{a}}$ On-tree prices for all sales methods as reported by the Florida Agricultural Statistics Service.

${ }^{b}$ Preliminary estimate by FASS for 2004-05 season.

${ }^{c}$ A management cost of $\$ 4.00$ per acre per month is included. Fixed costs such as taxes, debt service, and crop insurance are not included. 
Table 13.--Estimated annual per acre costs and returns and 5-year average costs and returns (adjusted to 2005 dollars) for a mature, red seedless grapefruit grove producing citrus for fresh fruit packing in the Southwest Florida area, 2000-01-2004-05

\begin{tabular}{|c|c|c|c|c|c|c|}
\hline Year & $\begin{array}{c}\text { Inflation } \\
\text { factor index } \\
\end{array}$ & $\begin{array}{c}\text { Adjusted } \\
\text { on-tree } \\
\text { price/box }\end{array}$ & Yield & $\begin{array}{l}\text { Gross } \\
\text { revenue }\end{array}$ & $\begin{array}{c}\text { Total specified } \\
\text { costs }^{\mathrm{b}}\end{array}$ & $\begin{array}{l}\text { Net return to land, } \\
\text { trees, and ownership }\end{array}$ \\
\hline & & & \multicolumn{4}{|c|}{ 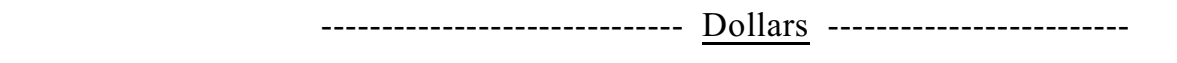 } \\
\hline 2000-01 & 117.9 & $\$ 2.69$ & 555 & $1,492.95$ & $1,076.18$ & 416.77 \\
\hline 2001-02 & 120.7 & $\$ 3.07$ & 555 & $1,703.85$ & $1,113.51$ & 590.34 \\
\hline 2002-03 & 114.6 & $\$ 2.86$ & 555 & $1,587.30$ & $1,124.31$ & 462.99 \\
\hline 2003-04 & 107.9 & $\$ 4.50$ & 555 & $2,497.50$ & 995.15 & $1,502.35$ \\
\hline 2004-05 & 100.0 & $\$ 14.27$ & 555 & $7,919.85$ & 997.74 & $6,922.11$ \\
\hline 5-yr. avg. & - & $\$ 5.48$ & 555 & $3,041.40$ & $1,061.38$ & $1,980.02$ \\
\hline
\end{tabular}

${ }^{a}$ Producer price index for each year adjusted to 2005 prices $(2005=100)$, with 2005 producer price index estimated to be 158.2. Producer price index for other years are: $2001=134.2 ; 2002=131.1 ; 2003=138.1$; and $2004=146.7$.

${ }^{\mathrm{b}}$ A management cost of $\$ 4.00$ per acre per month is included. Fixed costs such as taxes, debt service, and crop insurance are not included. (Refer to Table 12.) 


\section{REFERENCES}

1. Citrus Summary 2003-04. Florida Agricultural Statistics Service. Florida Agricultural Statistics. January 2005.

2. Muraro, Ronald P. "A Listing of Estimated Comparative Southwest Florida Citrus Production Costs Per Acre for 2004-05." Lake Alfred Citrus Research and Education Center (CREC) Report. Lake Alfred, FL: August 2005.

3. . Estimated Cost of Planting and Maintaining a Reset Citrus Tree through Three Years of Age." Lake Alfred CREC Report. Lake Alfred, FL: August 2005.

4. _. "A Listing of 2005 Custom Rates Reported by Sixteen Indian River and South Florida Citrus Caretakers." Lake Alfred CREC Report. Lake Alfred, FL: July 2005.

5. Savage, Zach. Citrus Yields Per Tree Age. Univ. of Fla. Agr. Ext. Ser. 60-8. Gainesville: 1960.

6. Timmer, L. W. (Ed.). 2005 Florida Citrus Pest Management Guide. Univ. of Fla. Coop. Ext. Svc. SP 43. Gainesville: Jan. 2005. 150 p.

7. Tucker, D. P. H., A. K. Alva, L. K. Jackson, and T. A. Wheaton (Eds.). Nutrition of Florida Citrus Trees. Univ. of Fla. Coop. Ext. Svc. SP 169. Gainesville: 1995. 61 pp. 
ADDENDA: $\quad$ Listing of Grove Care Programs for Southwest Florida Citrus Production for Both Round Oranges and Grapefruit ${ }^{\mathrm{a}}$

Table 1-A.

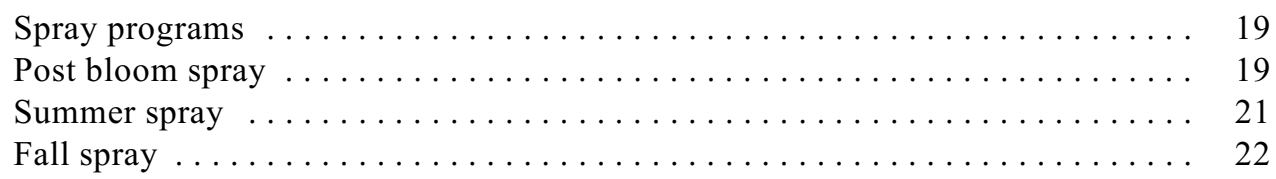

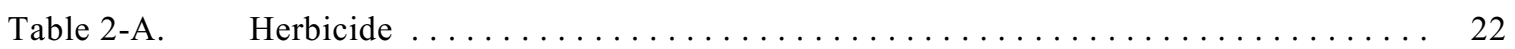

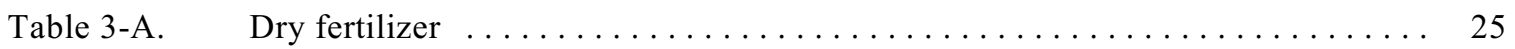

Table 4-A. $\quad$ Liquid fertilizer (Double boom application) $\ldots \ldots \ldots \ldots$

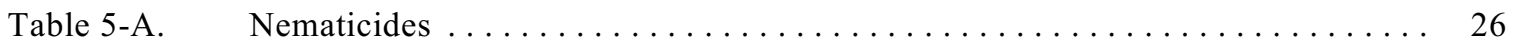

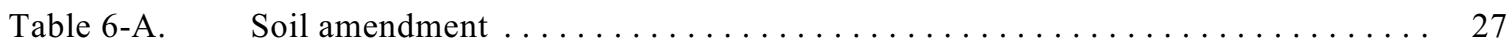

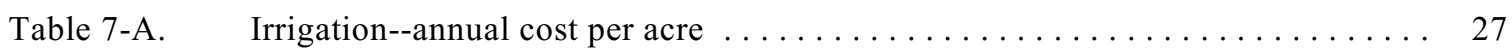

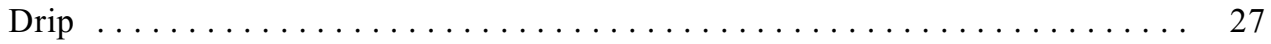

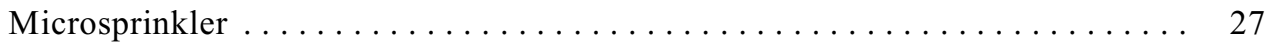

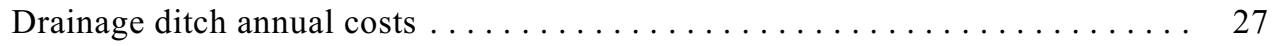

Table 8-A. A listing of 2005 custom rates reported by sixteen Indian River and South

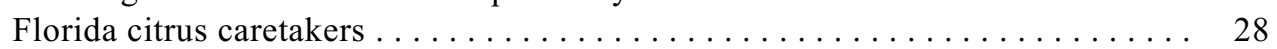

Table 9-A. 2005 summary of average chemical price estimates $\ldots \ldots \ldots 30$

Table 10-A. 2005 summary of average fertilizer price estimates $\ldots \ldots \ldots 32$

Table 11-A. A listing of estimated comparative Southwest Florida citrus production costs per

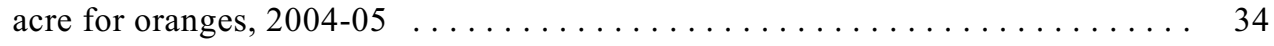

Table 12-A. A listing of estimated comparative Southwest Florida citrus production costs per

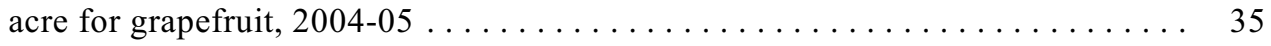

Table 13-A. Estimated cost of planting and maintaining a reset citrus tree through three years

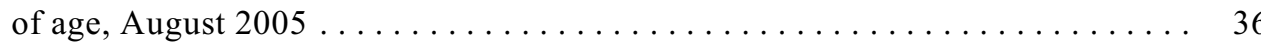

Table 14-A. Estimated average picking, roadsiding and hauling charges for Florida citrus,

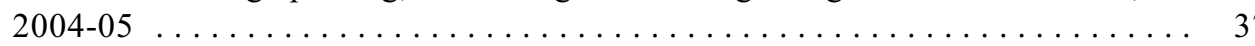

Table 15-A. Estimated average packing charges for Florida citrus, 2004-05 . . . . . . 38

Table 16-A. Historic prices for selected citrus varieties $\ldots \ldots \ldots \ldots \ldots$

Table 17-A. Debt which can be supported per $\$ 1,000.00$ annual payment capacity $\ldots \ldots \ldots 40$ Abbreviations for important chemicals are:
$\mathrm{B}=$ Boron
$\mathrm{Fe}=$ Iron
$\mathrm{Mn}=$ Manganese
$\mathrm{Zn}=\mathrm{Zinc}$
$\mathrm{Cu}=$ Copper
$\mathrm{Mg}=$ Magnesium
$\mathrm{N}=$ Nitrogen

${ }^{\text {a }}$ The costs in the ADDENDA represent an owner managed operation. Therefore, the 10 percent handling and supervision charge added to the material cost for a custom-managed operation is not included in the costs. 
Table 1-A.--Spray programs

\section{POST BLOOM SPRAY}

\begin{tabular}{|c|c|c|c|c|}
\hline \multirow[t]{7}{*}{ Spray Program \#1 } & $\underline{\text { Materials/Ingredients }}$ & $\begin{array}{l}\text { Amount } \\
\text { /Acre } \\
\end{array}$ & $\underline{\text { Cost/Acre }}$ & $\begin{array}{c}\text { Your } \\
\text { Cost/Acre } \\
\end{array}$ \\
\hline & $\mathrm{Cu}(50 \%$ metallic $)$ & $15 \mathrm{lbs}$ & $\$ 21.00$ & \\
\hline & $\mathrm{Zn}$ & $5 \mathrm{lbs}$ & 4.15 & \\
\hline & $\mathrm{Mn}$ & $10 \mathrm{lbs}$ & 3.20 & \\
\hline & B & $0.25 \mathrm{lb}$ & 1.17 & \\
\hline & $\begin{array}{l}\text { Ground Application } \\
\text { (PTO driven airblast) }\end{array}$ & 125 gals & $\underline{23.80}$ & \\
\hline & Total per Application & & $\$ 53.32$ & \\
\hline \multirow[t]{7}{*}{ Spray Program \#2 } & $\underline{\text { Materials/Ingredients }}$ & $\begin{array}{l}\text { Amount } \\
\text { /Acre } \\
\end{array}$ & $\underline{\text { Cost/Acre }}$ & $\begin{array}{c}\text { Your } \\
\text { Cost/Acre } \\
\end{array}$ \\
\hline & $\mathrm{Cu}(50 \%$ metallic $)$ & $10 \mathrm{lbs}$ & $\$ 14.00$ & \\
\hline & $\mathrm{Zn}$ & $5 \mathrm{lbs}$ & 4.15 & \\
\hline & $\mathrm{Mn}$ & $10 \mathrm{lbs}$ & 3.20 & \\
\hline & Micromite 25WP & $1.25 \mathrm{lbs}$ & 38.78 & \\
\hline & $\begin{array}{l}\text { Ground Application } \\
\text { (PTO driven airblast) }\end{array}$ & 100 gals & $\underline{24.24}$ & \\
\hline & Total per Application & & $\$ \underline{\underline{83.37}}$ & \\
\hline \multirow[t]{6}{*}{ Spray Program \#3 } & $\underline{\text { Materials/Ingredients }}$ & $\begin{array}{l}\text { Amount } \\
\text { /Acre } \\
\end{array}$ & $\underline{\text { Cost/Acre }}$ & $\begin{array}{c}\text { Your } \\
\text { Cost/Acre } \\
\end{array}$ \\
\hline & $\mathrm{Cu}(50 \%$ metallic $)$ & $7 \mathrm{lbs}$ & $\$ 9.80$ & \\
\hline & Agri-Mek & $10 \mathrm{ozs}$ & 44.10 & \\
\hline & Oil $97+\%$ & 3 gals & 6.63 & \\
\hline & $\begin{array}{l}\text { Ground Application } \\
\text { (PTO driven airblast) }\end{array}$ & 100 gals & $\underline{24.24}$ & \\
\hline & Total per Application & & $\$ \underline{\underline{84.77}}$ & \\
\hline \multirow[t]{6}{*}{ Spray Program \#4 } & $\underline{\text { Materials/Ingredients }}$ & $\begin{array}{l}\text { Amount } \\
\text { /Acre } \\
\end{array}$ & $\underline{\text { Cost/Acre }}$ & $\begin{array}{c}\text { Your } \\
\text { Cost/Acre } \\
\end{array}$ \\
\hline & $\mathrm{Cu}(50 \%$ metallic $)$ & $7 \mathrm{lbs}$ & $\$ 9.80$ & \\
\hline & Lorsban 4EC & 4 pts & 17.08 & \\
\hline & Hystop (pH Reducer) & $1 \mathrm{pt}$ & 2.30 & \\
\hline & $\begin{array}{l}\text { Ground Application } \\
\text { (PTO driven airblast) }\end{array}$ & 100 gals & $\underline{24.24}$ & \\
\hline & Total per Application & & $\$ 53.42$ & \\
\hline
\end{tabular}


Table 1-A.--Spray programs (cont'd.)

$\underline{\text { POST BLOOM SPRAY (cont'd.) }}$

\begin{tabular}{|c|c|c|c|c|}
\hline \multirow[t]{5}{*}{ Spray Program \#5 } & $\underline{\text { Materials/Ingredients }}$ & $\begin{array}{l}\text { Amount } \\
\text { /Acre }\end{array}$ & Cost/Acre & $\begin{array}{c}\text { Your } \\
\text { Cost/Acre } \\
\end{array}$ \\
\hline & Vendex 4L & $2 \mathrm{lbs}$ & $\$ 29.72$ & \\
\hline & $\mathrm{Cu}(50 \%$ metallic $)$ & $10 \mathrm{lbs}$ & 14.00 & \\
\hline & $\begin{array}{l}\text { Ground Application } \\
\text { (PTO driven airblast) }\end{array}$ & 250 gals & $\underline{27.95}$ & \\
\hline & Total per Application & & $\$ 71.67$ & \\
\hline \multirow[t]{6}{*}{ Spray Program \#6 } & $\underline{\text { Materials/Ingredients }}$ & $\begin{array}{l}\text { Amount } \\
\text { /Acre }\end{array}$ & Cost/Acre & $\begin{array}{c}\text { Your } \\
\text { Cost/Acre } \\
\end{array}$ \\
\hline & Micromite & $1.25 \mathrm{lbs}$ & $\$ 38.77$ & \\
\hline & $\mathrm{Cu}(50 \%$ metallic $)$ & $7 \mathrm{lbs}$ & 9.80 & \\
\hline & Oil $97+\%$ & 3 gals & 6.63 & \\
\hline & $\begin{array}{l}\text { Ground Application } \\
\text { (PTO driven airblast) }\end{array}$ & 250 gals & $\underline{27.95}$ & \\
\hline & Total per Application & & $\$ \underline{\underline{83.15}}$ & \\
\hline \multirow[t]{8}{*}{ Spray Program \#7 } & $\underline{\text { Materials/Ingredients }}$ & $\begin{array}{l}\text { Amount } \\
\text { /Acre } \\
\end{array}$ & $\underline{\text { Cost/Acre }}$ & $\begin{array}{c}\text { Your } \\
\text { Cost/Acre } \\
\end{array}$ \\
\hline & $\mathrm{Cu}(50 \%$ metallic $)$ & $7 \mathrm{lbs}$ & $\$ 9.80$ & \\
\hline & $\mathrm{Zn}$ & $5 \mathrm{lbs}$ & 4.15 & \\
\hline & $\mathrm{Mn}$ & $10 \mathrm{lbs}$ & 3.20 & \\
\hline & $\mathrm{B}$ & $0.25 \mathrm{lb}$ & 1.17 & \\
\hline & Micromite & $1.25 \mathrm{lbs}$ & 38.78 & \\
\hline & $\begin{array}{l}\text { Ground Application } \\
\text { (PTO driven airblast) }\end{array}$ & 250 gals & $\underline{27.95}$ & \\
\hline & Total per Application & & $\$ \underline{\underline{85.05}}$ & \\
\hline \multirow[t]{4}{*}{ Spray Program \#8 } & Materials/Ingredients & $\begin{array}{l}\text { Amount } \\
\text { /Acre } \\
\end{array}$ & Cost/Acre & $\begin{array}{c}\text { Your } \\
\text { Cost/Acre } \\
\end{array}$ \\
\hline & Vendex 4L & $2 \mathrm{lbs}$ & $\$ 29.72$ & \\
\hline & $\begin{array}{l}\text { Ground Application } \\
\text { (PTO driven airblast) }\end{array}$ & 125 gals & $\underline{23.80}$ & \\
\hline & Total per Application & & $\$ \underline{\underline{53.52}}$ & \\
\hline \multirow{4}{*}{$\begin{array}{c}\text { Spray Program \#9 } \\
\text { (Scale insects) }\end{array}$} & $\underline{\text { Materials/Ingredients }}$ & $\begin{array}{l}\text { Amount } \\
\text { /Acre } \\
\end{array}$ & Cost/Acre & $\begin{array}{c}\text { Your } \\
\text { Cost/Acre } \\
\end{array}$ \\
\hline & Lorsban 4EC & $5 \mathrm{pts}$ & $\$ 21.35$ & \\
\hline & $\begin{array}{l}\text { Ground Application } \\
\text { (engine driven airblast) }\end{array}$ & 500 gals & $\underline{36.60}$ & \\
\hline & Total per Application & & $\$ \underline{\underline{57.95}}$ & \\
\hline
\end{tabular}


Table 1-A.--Spray programs (cont'd.)

\section{SUMMER SPRAY}

\begin{tabular}{|c|c|c|c|c|}
\hline \multirow[t]{4}{*}{ Spray Program \#10 } & $\underline{\text { Materials/Ingredients }}$ & $\begin{array}{l}\text { Amount } \\
\text { /Acre }\end{array}$ & $\underline{\text { Cost/Acre }}$ & $\begin{array}{c}\text { Your } \\
\text { Cost/Acre } \\
\end{array}$ \\
\hline & $\begin{array}{l}\text { Oil } 97+\% \\
\mathrm{Cu}(50 \% \text { copper })\end{array}$ & $\begin{array}{l}10 \text { gals } \\
7 \mathrm{lbs}\end{array}$ & $\begin{array}{r}\$ 22.10 \\
9.80\end{array}$ & \\
\hline & $\begin{array}{l}\text { Ground Application } \\
\text { (PTO driven airblast) }\end{array}$ & 250 gals & $\underline{27.95}$ & \\
\hline & Total per Application & & $\$ \underline{\underline{59.85}}$ & \\
\hline \multirow[t]{8}{*}{ Spray Program \#11 } & $\underline{\text { Materials/Ingredients }}$ & $\begin{array}{l}\text { Amount } \\
\text { /Acre }\end{array}$ & $\underline{\text { Cost/Acre }}$ & $\begin{array}{c}\text { Your } \\
\text { Cost/Acre } \\
\end{array}$ \\
\hline & $\mathrm{Cu}(50 \%$ metallic $)$ & $7 \mathrm{lbs}$ & $\$ 9.80$ & \\
\hline & Oil $97+\%$ & 5 gals & 11.05 & \\
\hline & $\mathrm{Zn}$ & $5 \mathrm{lbs}$ & 4.15 & \\
\hline & $\mathrm{Mn}$ & $10 \mathrm{lbs}$ & 3.20 & \\
\hline & B & $0.25 \mathrm{lb}$ & 1.17 & \\
\hline & $\begin{array}{l}\text { Ground Application } \\
\text { (PTO driven airblast) }\end{array}$ & 125 gals & $\underline{23.80}$ & \\
\hline & Total per Application & & $\$ \underline{\underline{53.17}}$ & \\
\hline \multirow[t]{6}{*}{ Spray Program \#12 } & $\underline{\text { Materials/Ingredients }}$ & $\begin{array}{l}\text { Amount } \\
\text { /Acre } \\
\end{array}$ & $\underline{\text { Cost/Acre }}$ & $\begin{array}{c}\text { Your } \\
\text { Cost/Acre } \\
\end{array}$ \\
\hline & $\mathrm{Cu}(50 \%$ metallic $)$ & $7 \mathrm{lbs}$ & $\$ 9.80$ & \\
\hline & Oil $97+\%$ & 5 gals & 11.05 & \\
\hline & Agri-Mek & $10 \mathrm{ozs}$ & 44.10 & \\
\hline & $\begin{array}{l}\text { Ground Application } \\
\text { (PTO driven airblast) }\end{array}$ & 250 gals & $\underline{27.95}$ & \\
\hline & Total per Application & & $\$ \underline{\underline{92.90}}$ & \\
\hline \multirow[t]{6}{*}{ Spray Program \#13 } & $\underline{\text { Materials/Ingredients }}$ & $\begin{array}{l}\text { Amount } \\
\text { /Acre } \\
\end{array}$ & $\underline{\text { Cost/Acre }}$ & $\begin{array}{c}\text { Your } \\
\text { Cost/Acre } \\
\end{array}$ \\
\hline & Enable & $8 \mathrm{oz}$ & $\$ 14.40$ & \\
\hline & Oil $97+\%$ & 5 gals & 11.05 & \\
\hline & Micromite & $1.25 \mathrm{lbs}$ & 38.77 & \\
\hline & $\begin{array}{l}\text { Ground Application } \\
\text { (PTO driven airblast) }\end{array}$ & 125 gals & 23.80 & \\
\hline & Total per Application & & $\$ \underline{88.02}$ & \\
\hline
\end{tabular}


Table 1-A.--Spray programs (cont'd.)

SUMMER SPRAY (cont'd.)

Spray Program \#14

Materials/Ingredients

$\mathrm{Cu}(50 \%$ metallic $)$

Oil 97+\%

Ground Application

(PTO driven airblast)

Total per Application

\begin{tabular}{|c|c|c|}
\hline $\begin{array}{l}\text { Amount } \\
\text { /Acre }\end{array}$ & Cost/Acre & $\begin{array}{c}\text { Your } \\
\text { Cost/Acre } \\
\end{array}$ \\
\hline $7 \mathrm{lbs}$ & $\$ 9.80$ & \\
\hline 5 gals & 11.05 & \\
\hline 125 gals & 23.80 & \\
\hline & $\$ 44.65$ & \\
\hline
\end{tabular}

\section{$\underline{\text { FALL SPRAY }}$}

\begin{tabular}{|c|c|c|c|c|}
\hline \multirow[t]{4}{*}{ Spray Program \#15 } & $\underline{\text { Materials/Ingredients }}$ & $\begin{array}{l}\text { Amount } \\
\text { /Acre } \\
\end{array}$ & $\underline{\text { Cost/Acre }}$ & $\begin{array}{c}\text { Your } \\
\text { Cost/Acre } \\
\end{array}$ \\
\hline & Agri-Mek & 5 ozs & $\$ 22.05$ & \\
\hline & $\begin{array}{l}\text { Ground Application } \\
\text { (PTO driven airblast) }\end{array}$ & 125 gals & $\underline{23.80}$ & \\
\hline & Total per Application & & $\$ \underline{\underline{45.85}}$ & \\
\hline \multirow[t]{4}{*}{ Spray Program \#16 } & Materials/Ingredients & $\begin{array}{l}\text { Amount } \\
\text { /Acre }\end{array}$ & $\underline{\text { Cost/Acre }}$ & $\begin{array}{c}\text { Your } \\
\text { Cost/Acre } \\
\end{array}$ \\
\hline & Vendex 4L & $2 \mathrm{lbs}$ & $\$ 29.72$ & \\
\hline & Aerial Application & 15 GPA & 8.82 & \\
\hline & Total per Application & & $\$ \underline{\underline{38.54}}$ & \\
\hline \multicolumn{5}{|l|}{ Table 2-A.--Herbicide } \\
\hline \multirow{6}{*}{$\begin{array}{l}\text { Herbicide Program \#1 } \\
\text { (Strip/band) }\end{array}$} & $\underline{\text { Materials }}$ & $\begin{array}{c}\text { Amount/ } \\
\text { Treated Acre } \\
\end{array}$ & $\begin{array}{c}\text { Cost/ } \\
\text { Grove Acre }^{\mathrm{a}}\end{array}$ & $\begin{array}{l}\text { Your Cost/ } \\
\text { Grove Acre }\end{array}$ \\
\hline & Solicam 80DF & $3 \mathrm{lbs}$ & $\$ 21.36$ & \\
\hline & Karmex WP & $4 \mathrm{lbs}$ & 7.74 & \\
\hline & Roundup Ultra Max & 2 qts & 7.28 & \\
\hline & $\begin{array}{l}\text { Ground Application } \\
\text { (1 time) }\end{array}$ & & $\underline{9.06}$ & \\
\hline & Total for 1 Application & & $\$ \underline{\underline{45.44}}$ & \\
\hline \multirow{6}{*}{$\begin{array}{l}\text { Herbicide Program \#2 } \\
\text { (Strip/band) }\end{array}$} & $\underline{\text { Materials }}$ & $\begin{array}{c}\text { Amount/ } \\
\text { Treated Acre }\end{array}$ & $\begin{array}{c}\text { Cost/ } \\
\text { Grove Acre }^{\mathrm{a}}\end{array}$ & $\begin{array}{r}\text { Your Cost/ } \\
\text { Grove Acre } \\
\end{array}$ \\
\hline & Surflan A80 DF & $2 \mathrm{qts}$ & $\$ 20.42$ & \\
\hline & Simazine 4L & $4 \mathrm{qts}$ & 6.84 & \\
\hline & Roundup Ultra Max & $2 \mathrm{qts}$ & 7.28 & \\
\hline & $\begin{array}{l}\text { Ground Application } \\
\text { (1 time) }\end{array}$ & & $\underline{9.06}$ & \\
\hline & Total for 1 Application & & $\$ \underline{\underline{43.60}}$ & \\
\hline
\end{tabular}

${ }^{a}$ With respect to herbicide materials, Amount Per Grove Acre does not equal Amount Per Treated Acre shown on the label. Only a strip or band is being treated. In this report, it is assumed that only one-half of a surface acre is being treated. 
Table 2-A.--Herbicide (cont'd.)

\begin{tabular}{|c|c|c|c|c|}
\hline \multirow{5}{*}{$\begin{array}{l}\text { Herbicide Program \#3 } \\
\text { (Strip/band) }\end{array}$} & $\underline{\text { Materials }}$ & $\begin{array}{c}\text { Amount/ } \\
\text { Treated Acre } \\
\end{array}$ & $\begin{array}{c}\text { Cost } / \\
\text { Grove Acre }^{\mathrm{a}}\end{array}$ & $\begin{array}{r}\text { Your Cost/ } \\
\text { Grove Acre } \\
\end{array}$ \\
\hline & Karmex WP & $4 \mathrm{lbs}$ & $\$ 7.74$ & \\
\hline & Roundup Ultra Max & 2 qts & 7.28 & \\
\hline & $\begin{array}{l}\text { Ground Application } \\
\text { (1 time) }\end{array}$ & & $\underline{9.06}$ & \\
\hline & Total for 1 Application & & $\$ \underline{\underline{33.14}}$ & \\
\hline \multirow{6}{*}{$\begin{array}{l}\text { Herbicide Program \#4 } \\
\text { (Strip/band) }\end{array}$} & $\underline{\text { Materials }}$ & $\begin{array}{c}\text { Amount/ } \\
\text { Treated Acre } \\
\end{array}$ & $\begin{array}{c}\text { Cost } / \\
\text { Grove Acre }^{\mathrm{a}}\end{array}$ & $\begin{array}{r}\text { Your Cost/ } \\
\text { Grove Acre } \\
\end{array}$ \\
\hline & Solicam 80DF & $8 \mathrm{lbs}$ & $\$ 62.68$ & \\
\hline & Simazine 4L & 4 qts & 6.84 & \\
\hline & Roundup Ultra Max & 2 pts & 3.64 & \\
\hline & $\begin{array}{l}\text { Ground Application } \\
\text { (1 time) }\end{array}$ & & $\underline{9.06}$ & \\
\hline & Total for 1 Application & & $\$ \underline{\underline{82.22}}$ & \\
\hline \multirow{4}{*}{$\begin{array}{l}\text { Herbicide Program \#5 } \\
\text { (Strip/band) }\end{array}$} & $\underline{\text { Materials }}$ & $\begin{array}{c}\text { Amount/ } \\
\text { Treated Acre } \\
\end{array}$ & $\begin{array}{c}\text { Cost/ } \\
\text { Grove Acre }^{\mathrm{a}}\end{array}$ & $\begin{array}{r}\text { Your Cost/ } \\
\text { Grove Acre } \\
\end{array}$ \\
\hline & Roundup Ultra Max & 4 qts & $\$ 7.28$ & \\
\hline & $\begin{array}{l}\text { Ground Application } \\
\text { (1 time) }\end{array}$ & & $\underline{9.06}$ & \\
\hline & Total for 1 Application & & $\$ \underline{\underline{16.34}}$ & \\
\hline \multirow{5}{*}{$\begin{array}{l}\text { Herbicide Program \#6 } \\
\text { (Strip/band) }\end{array}$} & $\underline{\text { Materials }}$ & $\begin{array}{c}\text { Amount/ } \\
\text { Treated Acre } \\
\end{array}$ & $\begin{array}{c}\text { Cost/ } \\
\text { Grove Acre }^{\mathrm{a}}\end{array}$ & $\begin{array}{r}\text { Your Cost/ } \\
\text { Grove Acre } \\
\end{array}$ \\
\hline & Krovar I & $5 \mathrm{lbs}$ & $\$ 28.45$ & \\
\hline & Roundup Ultra Max & 2 qts & 7.28 & \\
\hline & $\begin{array}{l}\text { Ground Application } \\
\quad \text { (1 time) }\end{array}$ & & $\underline{9.06}$ & \\
\hline & Total for 1 Application & & $\$ \underline{\underline{44.79}}$ & \\
\hline \multirow{6}{*}{$\begin{array}{l}\text { Herbicide Program \#7 } \\
\text { (Strip/band) }\end{array}$} & $\underline{\text { Materials }}$ & $\begin{array}{c}\text { Amount/ } \\
\text { Treated Acre } \\
\end{array}$ & $\begin{array}{c}\text { Cost/ } \\
\text { Grove Acre }^{\mathrm{a}}\end{array}$ & $\begin{array}{r}\text { Your Cost/ } \\
\text { Grove Acre } \\
\end{array}$ \\
\hline & Princep (Caliber 90) & $4 \mathrm{lbs}$ & $\$ 6.58$ & \\
\hline & Hyvar X & $6 \mathrm{lbs}$ & 56.79 & \\
\hline & Adjuvant (Surfactant) & $2 \mathrm{pts}$ & 3.03 & \\
\hline & $\begin{array}{l}\text { Ground Application } \\
\text { (1 time) }\end{array}$ & & $\underline{9.06}$ & \\
\hline & Total for 1 Application & & $\$ \underline{\underline{75.46}}$ & \\
\hline
\end{tabular}


Table 2-A.--Herbicide (cont'd.)

\begin{tabular}{|c|c|c|c|c|}
\hline Herbicide Program \#8 & $\underline{\text { Materials }}$ & Treated Acre & $\underline{\text { Grove Acre }}^{a}$ & $\underline{\text { Grove Acre }}$ \\
\hline (Strip/band) & $\begin{array}{l}\text { Roundup Ultra Max } \\
\text { Princep (Caliber 90) }\end{array}$ & $\begin{array}{l}2 \mathrm{qts} \\
4 \mathrm{lbs}\end{array}$ & $\begin{array}{r}\$ 7.28 \\
6.58\end{array}$ & \\
\hline & $\begin{array}{l}\text { Ground Application } \\
\text { (1 time) }\end{array}$ & & $\underline{9.06}$ & \\
\hline & Total for 1 Application & & $\$ 22.92$ & \\
\hline Herbicide Program \#9 & Materials & $\begin{array}{c}\text { Amount/ } \\
\text { Treated Acre }\end{array}$ & $\begin{array}{c}\text { Cost/ } \\
\text { Grove Acre }^{\mathrm{a}}\end{array}$ & $\begin{array}{r}\text { Your Cost/ } \\
\text { Grove Acre } \\
\end{array}$ \\
\hline (Strip/band) & $\begin{array}{l}\text { Direx 4L } \\
\text { Solicam } \\
\text { Adjuvant (Surfactant) }\end{array}$ & $\begin{array}{l}3 \mathrm{qts} \\
3 \mathrm{lbs} \\
1 \mathrm{qt}\end{array}$ & $\begin{array}{r}\$ 6.21 \\
21.36 \\
3.04\end{array}$ & \\
\hline & $\begin{array}{l}\text { Ground Application } \\
\text { (1 time) }\end{array}$ & & $\underline{9.06}$ & \\
\hline & Total for 1 Application & & $\$ \underline{\underline{39.67}}$ & \\
\hline Herbicide Program \#10 & $\underline{\text { Materials }}$ & $\begin{array}{c}\text { Amount/ } \\
\text { Treated Acre } \\
\end{array}$ & $\begin{array}{c}\text { Cost/ } \\
\text { Grove Acre }^{\mathrm{a}}\end{array}$ & $\begin{array}{r}\text { Your Cost/ } \\
\text { Grove Acre } \\
\end{array}$ \\
\hline (Chemical mow) & Roundup Ultra & $1 \mathrm{pt}$ & $\$ 1.82$ & \\
\hline & $\begin{array}{l}\text { Ground Application } \\
\text { (1 time) }\end{array}$ & & $\underline{3.07}$ & \\
\hline & Total for 1 Application & & $\$ \underline{\underline{4.89}}$ & \\
\hline Herbicide Program \#11 & $\underline{\text { Materials }}$ & $\begin{array}{c}\text { Amount/ } \\
\text { Treated Acre } \\
\end{array}$ & $\begin{array}{c}\text { Cost/ } \\
\text { Grove Acre }^{\mathrm{a}}\end{array}$ & $\begin{array}{r}\text { Your Cost/ } \\
\text { Grove Acre } \\
\end{array}$ \\
\hline (Chemical mow) & Roundup Ultra & $1.5 \mathrm{pts}$ & $\$ 2.73$ & \\
\hline & $\begin{array}{l}\text { Ground Application } \\
\text { (1 time) }\end{array}$ & & $\underline{3.07}$ & \\
\hline & Total for 1 Application & & $\$ \underline{\underline{5.80}}$ & \\
\hline Herbicide Program \#12 & Materials & $\begin{array}{c}\text { Amount/ } \\
\text { Treated Acre }\end{array}$ & $\begin{array}{c}\text { Cost/ } \\
\text { Grove Acre }^{\mathrm{a}}\end{array}$ & $\begin{array}{r}\text { Your Cost/ } \\
\text { Grove Acre } \\
\end{array}$ \\
\hline (Spot treatment) & Roundup Ultra & 2 qts & $\$ 7.28$ & \\
\hline & $\begin{array}{l}\text { Ground Application } \\
\quad \text { (1 time) }\end{array}$ & & $\underline{4.60}$ & \\
\hline & Total for 1 Application & & $\$ \underline{11.88}$ & \\
\hline
\end{tabular}


Table 3-A.--Dry fertilizer

\begin{tabular}{|c|c|c|c|c|}
\hline Program \#1 & $\begin{array}{l}\text { Analysis/Material } \\
\text { Applied } \\
\end{array}$ & $\begin{array}{l}\text { Amount } \\
\text { /Acre }\end{array}$ & Cost/Acre & $\begin{array}{c}\text { Your } \\
\text { Cost/Acre } \\
\end{array}$ \\
\hline \multirow[t]{3}{*}{ (125 lbs N/Acre) } & $12-2-12-2.4 \mathrm{MgO}$ & $1042 \mathrm{lbs}$ & $\$ 105.25$ & \\
\hline & Application & 3 times & 16.59 & \\
\hline & Total for 3 Applications & & $\$ 121.84$ & \\
\hline \multirow{4}{*}{$\begin{array}{l}\text { Program } \# 2 \\
(150 \mathrm{lbs} \text { N/Acre })\end{array}$} & $\begin{array}{l}\text { Analysis/Material } \\
\text { Applied } \\
\end{array}$ & $\begin{array}{l}\text { Amount } \\
\text { /Acre } \\
\end{array}$ & Cost/Acre & $\begin{array}{c}\text { Your } \\
\text { Cost/Acre } \\
\end{array}$ \\
\hline & $15-2-15-2.4 \mathrm{MgO}$ & $1000 \mathrm{lbs}$ & $\$ 113.00$ & \\
\hline & Application & 3 times & 16.59 & \\
\hline & Total for 3 Applications & & $\$ \underline{\underline{129.59}}$ & \\
\hline \multirow{4}{*}{$\begin{array}{l}\text { Program \#3 } \\
\qquad(180 \mathrm{lbs} \text { N/Acre })\end{array}$} & $\begin{array}{l}\text { Analysis/Material } \\
\text { Applied }\end{array}$ & $\begin{array}{l}\text { Amount } \\
\text { /Acre }\end{array}$ & Cost/Acre & $\begin{array}{c}\text { Your } \\
\text { Cost/Acre } \\
\end{array}$ \\
\hline & $15-2-15-2.4 \mathrm{MgO}$ & $1200 \mathrm{lbs}$ & $\$ 135.60$ & \\
\hline & Application & 3 times & 16.59 & \\
\hline & Total for 3 Applications & & $\$ \underline{\underline{152.19}}$ & \\
\hline \multirow{4}{*}{$\begin{array}{l}\text { Program \#4 } \\
\text { (204 lbs N/Acre) }\end{array}$} & $\begin{array}{l}\text { Analysis/Material } \\
\text { Applied }\end{array}$ & $\begin{array}{l}\text { Amount } \\
\text { /Acre } \\
\end{array}$ & Cost/Acre & $\begin{array}{c}\text { Your } \\
\text { Cost/Acre } \\
\end{array}$ \\
\hline & $17-4-17-2.4 \mathrm{MgO}$ & $1200 \mathrm{lbs}$ & $\$ 142.80$ & \\
\hline & Application & 3 times & 16.59 & \\
\hline & Total for 3 Applications & & $\$ \underline{\underline{159.39}}$ & \\
\hline \multirow{4}{*}{$\begin{array}{l}\text { Program \#5 } \\
\text { (225 lbs N/Acre) }\end{array}$} & $\begin{array}{l}\text { Analysis/Material } \\
\text { Applied } \\
\end{array}$ & $\begin{array}{l}\text { Amount } \\
\text { /Acre }\end{array}$ & Cost/Acre & $\begin{array}{c}\text { Your } \\
\text { Cost/Acre } \\
\end{array}$ \\
\hline & $15-2-15-2.4 \mathrm{MgO}$ & $1500 \mathrm{lbs}$ & $\$ 169.50$ & \\
\hline & Application & 3 times & 16.59 & \\
\hline & Total for 3 Applications & & $\$ 186.09$ & \\
\hline
\end{tabular}


Table 4-A.--Liquid fertilizer (Double boom application)

\begin{tabular}{|c|c|c|c|c|}
\hline Program \#1 & $\begin{array}{c}\text { Analysis/Material } \\
\text { Applied } \\
\end{array}$ & $\begin{array}{l}\text { Amount } \\
\text { /Acre }\end{array}$ & Cost/Acre & $\begin{array}{c}\text { Your } \\
\text { Cost/Acre } \\
\end{array}$ \\
\hline \multirow[t]{3}{*}{ (180 lbs N/Acre) } & $10-0-10$ & $1800 \mathrm{lbs}$ & $\$ 151.85$ & \\
\hline & Double Boom Custom Application & 3 times & $\underline{37.65}$ & \\
\hline & Total for 3 Applications & & $\$ \underline{\underline{189.50}}$ & \\
\hline \multirow{4}{*}{$\begin{array}{l}\text { Program \#2 } \\
(180 \mathrm{lbs} \text { N/Acre })\end{array}$} & $\begin{array}{c}\text { Analysis/Material } \\
\text { Applied } \\
\end{array}$ & $\begin{array}{l}\text { Amount } \\
\text { /Acre }\end{array}$ & Cost/Acre & $\begin{array}{c}\text { Your } \\
\text { Cost/Acre } \\
\end{array}$ \\
\hline & $10-2-10$ & $1800 \mathrm{lbs}$ & $\$ 160.20$ & \\
\hline & Double Boom Custom Application & 3 times & 37.65 & \\
\hline & Total for 3 Applications & & $\$ \underline{\underline{197.85}}$ & \\
\hline \multirow{6}{*}{$\begin{array}{l}\text { Program \#3 } \\
(180 \mathrm{lbs} \text { N/Acre })\end{array}$} & $\begin{array}{c}\text { Analysis/Material } \\
\text { Applied } \\
\end{array}$ & $\begin{array}{l}\text { Amount } \\
\text { /Acre }\end{array}$ & Cost/Acre & $\begin{array}{c}\text { Your } \\
\text { Cost/Acre } \\
\end{array}$ \\
\hline & $10-0-10$ & $1800 \mathrm{lbs}$ & $\$ 151.85$ & \\
\hline & Solicam 80DF & $3 \mathrm{lbs}$ & 21.36 & \\
\hline & Karmex WP & $4 \mathrm{lbs}$ & 7.74 & \\
\hline & Double Boom Custom Application & 3 times & 37.65 & \\
\hline & Total for 3 Applications & & $\$ 217.95$ & \\
\hline
\end{tabular}

Table 5-A.--Nematicides

\begin{tabular}{|c|c|c|c|c|}
\hline \multirow[t]{4}{*}{ Program \#1 } & $\begin{array}{c}\text { Analysis/Material } \\
\text { Applied } \\
\end{array}$ & $\begin{array}{l}\text { Amount } \\
\text { /Acre }\end{array}$ & $\underline{\text { Cost/Acre }}$ & $\begin{array}{c}\text { Your } \\
\text { Cost/Acre } \\
\end{array}$ \\
\hline & Temik 15G & $33 \mathrm{lbs}$ & $\$ 105.60$ & \\
\hline & Application & & 11.34 & \\
\hline & Total per Application & & $\$ 116.94$ & \\
\hline
\end{tabular}


Table 6-A.--Soil amendment

\begin{tabular}{|c|c|c|c|c|}
\hline \multirow{5}{*}{$\begin{array}{l}\text { Program \#1 } \\
\text { (Every } 3 \text { years) }\end{array}$} & $\begin{array}{l}\text { Analysis/Material } \\
\text { Applied } \\
\end{array}$ & $\begin{array}{l}\text { Amount } \\
\text { /Acre } \\
\end{array}$ & $\underline{\text { Cost/Acre }}$ & $\begin{array}{c}\text { Your } \\
\text { Cost/Acre } \\
\end{array}$ \\
\hline & Dolomite (Delivered) & 1 ton & $\$ 36.05$ & \\
\hline & Application & 1 time & $\underline{7.61}$ & \\
\hline & Total for 1 Application & & $\$ \underline{\underline{43.66}}$ & \\
\hline & (Average $1 / 3$ Ton Applied/Yr) & & $\$ 14.56$ & \\
\hline \multirow{4}{*}{$\begin{array}{l}\text { Program \#2 } \\
\text { (Every year) }\end{array}$} & $\begin{array}{l}\text { Analysis/Material } \\
\text { Applied } \\
\end{array}$ & $\begin{array}{l}\text { Amount } \\
\text { /Acre } \\
\end{array}$ & $\underline{\text { Cost/Acre }}$ & $\begin{array}{c}\begin{array}{c}\text { Your } \\
\text { Cost/Acre }\end{array} \\
\end{array}$ \\
\hline & Dolomite (Delivered) & $1000 \mathrm{lbs}$ & $\$ 18.03$ & \\
\hline & Application & & 7.61 & \\
\hline & Total per Application & & $\$ 25.64$ & \\
\hline
\end{tabular}

Table 7-A.--Irrigation--annual cost per acre

\begin{tabular}{|c|c|c|c|c|}
\hline$\underline{\text { DRIP }}$ & $\underline{\text { Program \#1 }}$ & $\begin{array}{c}\text { Your } \\
\text { Cost/Acre } \\
\end{array}$ & $\underline{\text { Program \#2 }}$ & $\begin{array}{c}\text { Your } \\
\text { Cost/Acre }\end{array}$ \\
\hline Operating & $\begin{array}{l}\text { (Electric) } \\
\$ 62.10\end{array}$ & & $\begin{array}{l}\text { (Diesel) } \\
\$ 55.87\end{array}$ & \\
\hline Maintenance of System & 44.04 & & 43.82 & \\
\hline Total Cash Expenses & $\$ 106.14$ & & $\$ 99.69$ & \\
\hline Fixed Depreciation Expense & $\underline{42.35}$ & & $\underline{45.25}$ & \\
\hline Total Cash and Fixed Expenses & $\$ \underline{\underline{148.49}}$ & & $\$ \underline{\underline{144.94}}$ & \\
\hline MICROSPRINKLER & Program \#3 & $\begin{array}{c}\text { Your } \\
\text { Cost/Acre }\end{array}$ & Program \#4 & $\begin{array}{c}\text { Your } \\
\text { Cost/Acre }\end{array}$ \\
\hline Operating & $\begin{array}{l}\text { (Electric) } \\
\$ 70.60\end{array}$ & & $\begin{array}{l}\text { (Diesel) } \\
\$ 59.44\end{array}$ & \\
\hline Maintenance of System & $\underline{49.08}$ & & $\underline{50.17}$ & \\
\hline Total Cash Expenses & $\$ 119.68$ & & $\$ 109.61$ & \\
\hline Fixed Depreciation Expense & 52.94 & & 56.56 & \\
\hline Total Cash and Fixed Expenses & $\$ 172.62$ & & $\$ \underline{\underline{166.17}}$ & \\
\hline \multicolumn{2}{|c|}{ DRAINAGE DITCH ANNUAL COSTS } & & $\underline{\text { Program } \# 5}$ & $\begin{array}{c}\text { Your } \\
\text { Cost/Acre }\end{array}$ \\
\hline \multicolumn{3}{|c|}{ Ditches/Canals Maintenance $(\$ 45.17$ /acre $\div 3$ years $)$} & $\$ 15.06$ & \\
\hline \multicolumn{3}{|c|}{ Weed Control in Ditches/Canals } & 14.19 & \\
\hline \multicolumn{3}{|c|}{ Water Control: In/Out of Ditches and Canals } & $\underline{13.21}$ & \\
\hline Total & & & $\$ \underline{\underline{42.36}}$ & \\
\hline
\end{tabular}


Table 8-A.--A listing of 2005 custom rates reported by sixteen Indian River and South Florida citrus caretakers

\begin{tabular}{|c|c|c|c|c|c|}
\hline Grove Practice & \multicolumn{3}{|c|}{ Range of Rate } & Average & Comments \\
\hline \multicolumn{6}{|l|}{ CULTIVATION AND EQUIPMENT: } \\
\hline Labor & Hour & $\$ 9.50-$ & $\$ 17.50$ & $\$ 13.56$ & Plus transportation and equipment \\
\hline Mechanic Labor & Hour & $30.00-$ & 50.00 & 39.56 & Labor and service truck \\
\hline Rotovate & Hour & $33.00-$ & 40.00 & 37.75 & \\
\hline Disc 7-8' & Hour & $27.50-$ & 38.50 & 33.00 & \\
\hline Disc 10-12' & Hour & $32.00-$ & 40.00 & 35.84 & \\
\hline Mow: 7-8' & Hour & $27.50-$ & 35.00 & 31.14 & \\
\hline $9-10^{\prime}$ & Hour & $31.00-$ & 38.50 & 33.97 & \\
\hline $9-10^{\prime}$ & Acre & $9.00-$ & 11.00 & 9.97 & \\
\hline $15-16^{\prime}$ & Hour & $35.00-$ & 41.25 & 39.85 & $\$ 9.00 /$ acre \\
\hline V-Mower & Hour & $33.00-$ & 35.00 & 34.34 & \\
\hline Herbicide $^{z}$ (Strip/Band-Single Boom) & Hour & $30.00-$ & 32.00 & 31.33 & Plus materials \\
\hline Herbicide $^{\mathrm{z}}$ (Strip/Band-Single Boom) & Acre & $13.00-$ & 13.75 & 13.39 & Plus materials; $\$ 35.00 /$ hour \\
\hline Herbicide $^{\mathrm{z}}$ (Strip/Band-Double Boom) & Acre & $12.00-$ & 14.00 & 12.75 & Plus materials \\
\hline Herbicide $^{\mathrm{z}}$ (Chemical Mow) & Acre & $2.50-$ & 3.50 & 3.07 & Plus materials \\
\hline Temik $^{z}$ & Acre & $10.50-$ & 12.50 & 11.34 & Plus materials \\
\hline Plow & Hour & $32.50-$ & 38.50 & 34.67 & \\
\hline Backhoe & Hour & $45.00-$ & 47.50 & 45.70 & \\
\hline Middle Buster & Hour & - & - & 38.50 & With tractor and driver \\
\hline Mound Builder & Hour & $31.00-$ & 38.50 & 34.84 & With tractor and driver \\
\hline Grader Blade & Hour & $28.00-$ & 38.50 & 33.60 & Tractor/blade and driver \\
\hline Water Truck with Driver & Hour & $30.00-$ & 35.00 & 32.75 & \\
\hline Pickup Truck with Driver & Hour & $28.00-$ & 35.00 & 31.67 & Average miles traveled per year: Pick-up truck - 21,298 miles \\
\hline Flatbed/Transport Truck with Driver & Hour & $35.00-$ & 50.00 & 45.00 & \\
\hline Tractor with Driver & Hour & $28.00-$ & 35.00 & 31.69 & \\
\hline ATV with Driver & Hour & $20.00-$ & 25.00 & 23.25 & \\
\hline
\end{tabular}

\section{$\underline{\text { SPRAYING: }}{ }^{2}$}

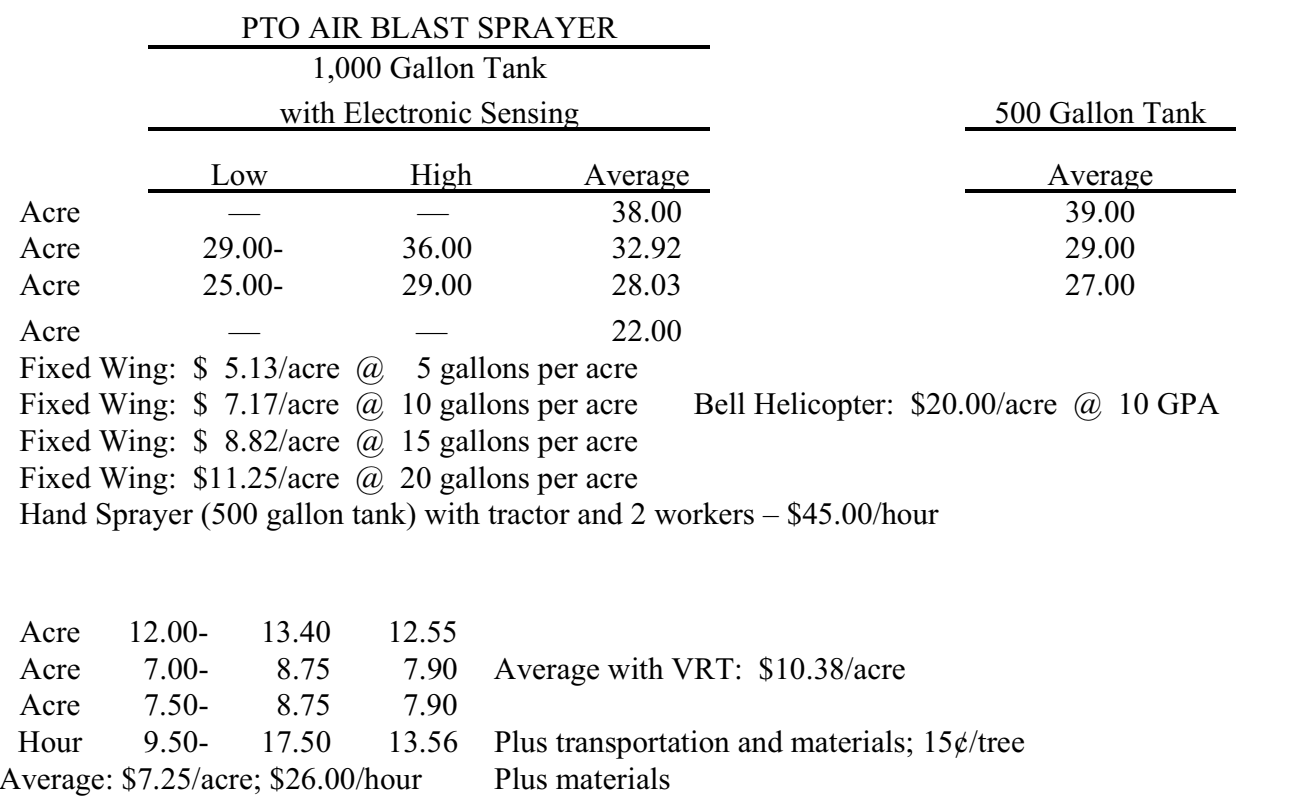


Table 8-A.--A listing of 2005 custom rates reported by sixteen Indian River and South Florida citrus caretakers (cont'd.)

\begin{tabular}{|c|c|c|c|c|c|}
\hline Grove Practice & \multicolumn{2}{|c|}{ Range of Rate } & $\begin{array}{l}\text { of Rate } \\
\text { orted }\end{array}$ & $\begin{array}{c}\text { Average } \\
\text { Rate }^{\mathrm{y}}\end{array}$ & Comments \\
\hline \multicolumn{6}{|l|}{ IRRIGATION: } \\
\hline Ditch Mower & Hour & $\$ 32.00-$ & $\$ 44.50$ & $\$ 36.20$ & \\
\hline Water Furrow Disc & Hour & $30.00-$ & 38.50 & 34.67 & \\
\hline Water Furrow Cleaner & Hour & $35.00-$ & 38.50 & 36.34 & \\
\hline Water Furrow Shaper (Non-Laser Control) & Hour & - & - & 65.00 & \\
\hline Water Furrow Shaper (Laser Control) & Hour & - & - & 80.00 & \\
\hline Rotary Ditcher or Auger & Hour & $33.00-$ & 38.50 & 35.50 & \\
\hline Microsprinkler/Drip Irrigation Maintenance & cre/Month & $3.50-$ & 4.75 & 4.25 & Check \& repair system; parts extra \\
\hline \multicolumn{6}{|l|}{ REMOVING TREES: } \\
\hline Front-end Loader & Hour & $\$ 50.00-$ & $\$ 65.00$ & $\$ 56.79$ & Avg. range 3-15 trees per hour \\
\hline Tree Shearing (Cutting Tree at Ground Level) & Hour & $50.00-$ & 65.00 & 56.25 & Avg. range 5-20 trees per hour \\
\hline Prepare Site for Replanting & Tree & $\$ 0.2$ & $-\$ 1.00$ & & \\
\hline \multicolumn{6}{|l|}{ PRUNING: } \\
\hline \multicolumn{6}{|l|}{$\overline{\text { Hedging: }}$} \\
\hline Single Side (Tractor Mounted) & Hour & $\$-$ & $\$-$ & $\$ 55.00$ & \\
\hline Double Side (Tractor Pulled) & Hour & - & - & 65.00 & \\
\hline Double Side (Self Propelled) & Hour & $250.00-$ & 265.00 & 257.50 & 8 to $20 \mathrm{~A} / \mathrm{H}$ depending on wood size; $\$ 14 / \mathrm{A}$ annual cut \\
\hline Double Side Rotary (Self Propelled) ${ }^{\mathrm{x}}$ & Hour & - & - & 185.00 & 5 to $15 \mathrm{~A} / \mathrm{H}$ bed tops only; add $25 \%$ for furrows only \\
\hline \multicolumn{6}{|l|}{ Topping: } \\
\hline Double Sided Topper (Self Propelled) & Hour & $265.00-$ & 285.00 & 275.00 & Avg. $8-15$ ac depending on wood size type of cut $\$ 30 /$ acre \\
\hline Topping Self Propelled & Hour & - & - & 150.00 & \\
\hline Limb Lifter/Tree Skirt Trimmer & Acre & - & - & 14.00 & 3 to 5 acres/hour \\
\hline $\begin{array}{l}\text { Limb Lifter/Tree Skirt Trimmer (Double Sided Rotary) } \\
\text { Removing Brush: }\end{array}$ & Hour & - & - & 120.00 & 6 to 20 acres/hour \\
\hline Haul Brush out of Grove (Front-End Loader) & Hour & $55.00-$ & 65.00 & 59.25 & \\
\hline Mow/Chop Brush & Hour & $32.00-$ & 40.00 & 34.60 & \\
\hline \multicolumn{6}{|l|}{ OTHER CUSTOM RATES: } \\
\hline$\overline{\text { Install Tree Wraps }}$ & $15 \not-50 \phi$ & $/$ tree deper & ding on ty & pe of wrap & and number of trees; Annual maintenance cost: $35 \phi /$ tree \\
\hline Plant Trees (Solid Set) & Tree & $\$ 0.90-$ & $\$ 1.75$ & $\$ 1.32$ & Varies as to density \\
\hline Plant Trees (Resets) & Tree & $2.00-$ & 2.50 & 2.17 & Varies as to the number of resets \\
\hline Travel/Setup Charge & Hour & - & - & 22.62 & \\
\hline \multicolumn{6}{|l|}{ Grove Management Charge/Month: } \\
\hline Supervising Grove Care Operations & Acre & $3.00-$ & 7.50 & 5.15 & In addition to caretaking charges \\
\hline Handling Fruit Marketing & $\$ 0.10-\$ 0$ & $0.25 / \mathrm{box}-$ & For Superv & ising and $\mathrm{N}$ & Marketing fruit \\
\hline Supervising/Handling Chemicals/Fertilizer & $5 \%$ to 15 & $5 \%$ of mat & rials cost & & \\
\hline $\begin{array}{l}\text { Charge for personnel to oversee harvesting } \\
\text { operations and coordinate harvest in different } \\
\text { blocks/groves and keeping of harvesting labor } \\
\text { compliance records. }\end{array}$ & Box & $\$ 0.05-$ & $\$ 0.25$ & $\$ 0.15$ & \\
\hline Consulting & Hour & $\$ 125.00-$ & $\$ 200.00$ & $\$ 150.00$ & Horticultural Evaluation and/or Financial Analysis/prospectus. \\
\hline Total Reported Acreage Provided Grove Service to: & Acre & $1,000-$ & 14,000 & 4,870 & Total acres reporting: 48,700 \\
\hline
\end{tabular}

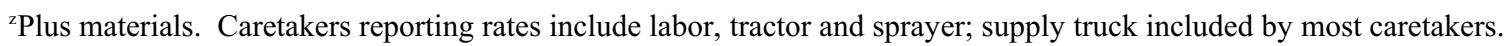

${ }^{y}$ Calculated by dividing the total number of caretakers reporting a grove practice rate into the sum reported. Unless otherwise stated, labor included with all charges.

${ }^{\mathrm{x}}$ Low acres is for 2 years regrowth hedging; high acres is for annual maintenance hedging.

Source: Ronald P. Muraro, Extension Farm Management Economist, Lake Alfred CREC, July 2005. 
Table 9-A.--2005 summary of average chemical price estimates

\begin{tabular}{|c|c|c|c|c|}
\hline \multicolumn{2}{|l|}{ Item } & \multirow{2}{*}{$\frac{\text { Unit }}{\text { gal. }}$} & \multirow{2}{*}{$\begin{array}{c}\begin{array}{c}\text { Average } \\
\text { Price }\end{array} \\
218.12\end{array}$} & \multirow[t]{2}{*}{$\begin{array}{c}\text { Your Price } \\
(2005)\end{array}$} \\
\hline Fungicides: & Abound EC & & & \\
\hline & Aliette 80WP & lb. & 11.59 & \\
\hline & Basic Copper Sulfate & lb. & 1.40 & \\
\hline & Copper (Kocide 101) & lb. & 1.80 & \\
\hline & Copper (Kocide 2000) & lb. & 2.33 & \\
\hline & Copper (Champ II Flowable) & gal. & 22.55 & \\
\hline & Cuprofix Disperss & lb. & 1.75 & \\
\hline & Nu-Cop 50 DF & lb. & 1.88 & \\
\hline & Enable & gal. & 57.55 & \\
\hline & Gem 25 & 40 ozs. & 120.59 & \\
\hline & Headline EC & gal. & 206.13 & \\
\hline & Oil - 435 or 455 & gal. & 2.21 & \\
\hline & Oil - 470 (Bio-lever) & gal. & 2.46 & \\
\hline & Ridomil Gold EC & gal. & 649.15 & \\
\hline & Safe-T-Oil & gal. & 3.15 & \\
\hline & Topsin & lb. & 14.08 & \\
\hline \multicolumn{5}{|c|}{ Insecticides/Nematicides: } \\
\hline & Admire $2 \mathrm{~F}$ & gal. & 520.28 & \\
\hline & Agri-Mek (0.15EC) & gal. & 563.52 & \\
\hline & Award Fire Ant Bait & lb. & 9.01 & \\
\hline & Bio-Vector & gal. & 412.50 & \\
\hline & Carbaryl 4L & gal. & 27.25 & \\
\hline & Carbaryl 80S & $\mathrm{lb}$. & 4.47 & \\
\hline & Chlorpyrifos 4E & gal. & 57.26 & \\
\hline & Danitol & gal. & 147.58 & \\
\hline & Guthion 2L & gal. & 32.48 & \\
\hline & Guthion 50WP & lb. & 10.07 & \\
\hline & Imidan 70W (Diaprepes) & lb. & 8.25 & \\
\hline & Lorsban 4EC & gal. & 34.15 & \\
\hline & Lorsban $15 \mathrm{G}$ & lb. & 1.72 & \\
\hline & Malathion 5 EC & gal. & 25.18 & \\
\hline & Micromite $80 \mathrm{WG}$ & gal. & 87.95 & \\
\hline & Microthiol & lb. & 0.70 & \\
\hline & Nexter 75WP & lb. & 89.56 & \\
\hline & Provado 1.6 F (nursery) & gal. & 417.75 & \\
\hline & Sevin $80 \mathrm{~S}$ & lb. & 5.17 & \\
\hline & Sevin XLR & gal. & 30.96 & \\
\hline & Spintor $2 \mathrm{~S} \mathrm{C}$ & gal. & 492.50 & \\
\hline & Sulphur 6F & gal. & 4.00 & \\
\hline & Temik 15G & lb. & 3.20 & \\
\hline & Vendex 50W & lb. & 14.86 & \\
\hline & Vydate & gal. & 56.28 & \\
\hline
\end{tabular}


Table 9-A.--2005 summary of average chemical price estimates (cont'd.)

\begin{tabular}{|c|c|c|c|c|}
\hline \multicolumn{2}{|l|}{ Item } & \multirow{2}{*}{$\frac{\text { Unit }}{\text { gal. }}$} & \multirow{2}{*}{$\begin{array}{c}\begin{array}{c}\text { Average } \\
\text { Price }\end{array} \\
48.39\end{array}$} & \multirow[t]{2}{*}{$\begin{array}{c}\text { Your Price } \\
(2005) \\
\end{array}$} \\
\hline Herbicides: & Aqua Master & & & \\
\hline & Diuron 4L & gal. & 16.04 & \\
\hline & Direx 4L & gal. & 16.50 & \\
\hline & Direx $80 \mathrm{DF}$ & lb. & 3.87 & \\
\hline & Fusilade DX 2E & gal. & 131.14 & \\
\hline \multicolumn{5}{|c|}{ Glyphosate: } \\
\hline & Glyphomax Plus & gal. & 18.22 & \\
\hline & Roundup (Original) & gal. & 23.60 & \\
\hline & Roundup - Ultra Max & gal. & 29.12 & \\
\hline & Roundup Weather Max & gal. & 50.16 & \\
\hline & Roundup Original Max & gal. & 43.50 & \\
\hline & Touchdown & gal. & 37.05 & \\
\hline & Gramoxone E (Paraquat) & gal. & 37.53 & \\
\hline & Hyvar X 80 WP & lb. & 18.93 & \\
\hline & Karmex 80 DF & lb. & 3.87 & \\
\hline & Krovar I & lb. & 11.38 & \\
\hline & Landmaster II & gal. & 18.66 & \\
\hline & Mandate 2E & gal. & 166.09 & \\
\hline & Pendimax & gal. & 24.37 & \\
\hline & Poast Plus 1.0 EC & gal. & 52.50 & \\
\hline & Princep (Caliber 90) & lb. & 3.29 & \\
\hline & Princep 4L & gal. & 14.51 & \\
\hline & Prowl & gal. & 22.12 & \\
\hline & Simazine $90 \mathrm{DF}$ & lb. & 2.80 & \\
\hline & Simazine 4L & gal. & 13.66 & \\
\hline & Solicam 80 DF & lb. & 14.24 & \\
\hline & Simtrol & & 19.00 & \\
\hline & Surflan & gal. & 81.64 & \\
\hline \multicolumn{5}{|c|}{ Growth Regulators: } \\
\hline & Citrus Fix & gal. & 494.00 & \\
\hline & Pro-Gibb 3.91\% & 20 oz. bottle & 33.16 & \\
\hline & Tree-Hold & gal. & 79.17 & \\
\hline \multicolumn{5}{|c|}{ Other Spray Materials: } \\
\hline & Borates $(15 \%)$ & lb. & 0.70 & \\
\hline & Manganese (32\%) & lb. & 0.32 & \\
\hline & Zinc $(78 \%)$ & lb. & 0.83 & \\
\hline & Adjuvant (Surfactant) & gal. & 23.59 & \\
\hline
\end{tabular}

SOURCE: Ronald P. Muraro, Extension Farm Management Economist, University of Florida, IFAS, CREC, Lake Alfred, Florida, August 2005. 
Table 10-A.--2005 summary of average fertilizer price estimates

\begin{tabular}{|c|c|c|c|}
\hline Item & Unit & $\begin{array}{c}\text { Average } \\
\text { Price } \\
\end{array}$ & $\begin{array}{c}\text { Your Price } \\
(2005) \\
\end{array}$ \\
\hline \multicolumn{4}{|l|}{ FERTILIZER (FOB Price @ Plant) } \\
\hline & & $\$$ & \\
\hline \multicolumn{4}{|l|}{$\underline{\text { Dry Mix (Bulk) }}$} \\
\hline $17-0-17-3_{\mathrm{Mg}}$ & ton & 238.82 & \\
\hline $17-4-17-2.4_{\mathrm{Mg}}$ & ton & 243.35 & \\
\hline $16-0-16$ & ton & 218.35 & \\
\hline $16-0-16-4_{\mathrm{Mg}}$ & ton & 239.49 & \\
\hline $16-2-16-3_{\mathrm{Mg}}$ & ton & 240.45 & \\
\hline $15-2-15-2.4_{\mathrm{Mg}}$ & ton & 224.47 & \\
\hline $12-2-12-2.4_{\mathrm{Mg}}$ & ton & 201.02 & \\
\hline $8-8-8 \mathrm{w} /$ minors* & ton & 182.90 & \\
\hline $8-4-8 \mathrm{w} /$ minors* & ton & 170.29 & \\
\hline $8-2-8 \mathrm{w} / \mathrm{minors} *$ & ton & 162.12 & \\
\hline 6-6-6 w/minors* & ton & 159.99 & \\
\hline \multicolumn{4}{|l|}{ Liquid Mix (Bulk) } \\
\hline $8-2-8$ & ton & 151.53 & \\
\hline $8-4-8$ & ton & 159.73 & \\
\hline $9-3-9$ & ton & 166.33 & \\
\hline $9-4-9$ & ton & 172.47 & \\
\hline $10-0-10$ & ton & 166.62 & \\
\hline $10-2-10$ & ton & 176.25 & \\
\hline $12-0-6$ & ton & 166.89 & \\
\hline $12-3-6$ & ton & 180.25 & \\
\hline 7-0-0-6 (Magnesium Nitrate) & ton & 218.00 & \\
\hline
\end{tabular}

*With organic nitrogen, the price averaged $25 \%$ higher. 
Table 10-A.--2005 summary of average fertilizer price estimates (cont'd.)

\begin{tabular}{|c|c|c|c|}
\hline Item & Unit & $\begin{array}{c}\text { Average } \\
\text { Price }\end{array}$ & $\begin{array}{c}\text { Your Price } \\
(2005) \\
\end{array}$ \\
\hline \multicolumn{4}{|l|}{ Other Fertilizer Materials (Bulk) } \\
\hline Ammonium Nitrate (21\% N Liquid) & ton & 179.88 & \\
\hline Ammonium Nitrate (33.5\% N Dry) & ton & 259.38 & \\
\hline Ammonium Sulfate $(21 \% \mathrm{~N})$ & ton & 152.94 & \\
\hline Calcium Nitrate $(19 \% \mathrm{Ca}, 15.5 \% \mathrm{~N})$ & ton & 288.13 & \\
\hline Dolomite (at mine-- $49 \% \mathrm{CaCO}_{3}, 36 \% \mathrm{MgCO}_{3}$ ) & ton & 19.75 & \\
\hline Muriate of Potash $\left(60 \% \mathrm{~K}_{2} \mathrm{O}\right)$ & ton & 242.29 & \\
\hline Potassium Nitrate $\left(14 \% \mathrm{~N} ; 46 \% \mathrm{~K}_{2} \mathrm{O}\right)$ & ton & 453.57 & \\
\hline Sul-Po-Mag (SPM--21.9\% $\left.\mathrm{K}_{2} \mathrm{O}\right)$ & ton & 202.43 & \\
\hline Super Phosphate $\left(20 \% \mathrm{P}_{2} \mathrm{O}_{5}\right)$ & ton & 214.25 & \\
\hline Triple Superphosphate $\left(48 \% \mathrm{P}_{2} \mathrm{O}_{5}\right)$ & ton & 242.92 & \\
\hline Average Delivery Cost & ton & 14.32 & \\
\hline \multicolumn{4}{|l|}{$\underline{\text { Foliar Macronutrients }}$} \\
\hline Phos Might 0-22-20 & gal. & 24.29 & \\
\hline Nutriphite Magnum 2-40-16 & gal. & 35.00 & \\
\hline MKP (0-52-34) (Mono-Potassium Phosphate) & lb. & 0.80 & \\
\hline RSA ActaPhos 0-28-25 & gal. & 18.00 & \\
\hline Peter's 20-20-20 Foliar & lb. & 0.54 & \\
\hline $\mathrm{MZF}$ & gal. & 6.53 & \\
\hline \multicolumn{4}{|l|}{$\underline{\text { Slow Release Nitrogen (SRN) }}$} \\
\hline \multicolumn{4}{|l|}{ CitriBlen } \\
\hline $15-3-19$ & ton & 245.15 & \\
\hline $17-5-12$ & ton & 237.50 & \\
\hline $18-6-11$ & ton & 243.80 & \\
\hline Sulfur Coated Urea (SCU) & ton & 586.80 & \\
\hline Agriform 20-10-5 (500 tablets/box) & box & 40.00 & \\
\hline
\end{tabular}

SOURCE: Ronald P. Muraro, Extension Farm Management Economist, University of Florida, IFAS, CREC, Lake Alfred, Florida, August 2005. 
Table 11-A.--A listing of estimated comparative Southwest Florida citrus production costs per acre for oranges, 2004-2005

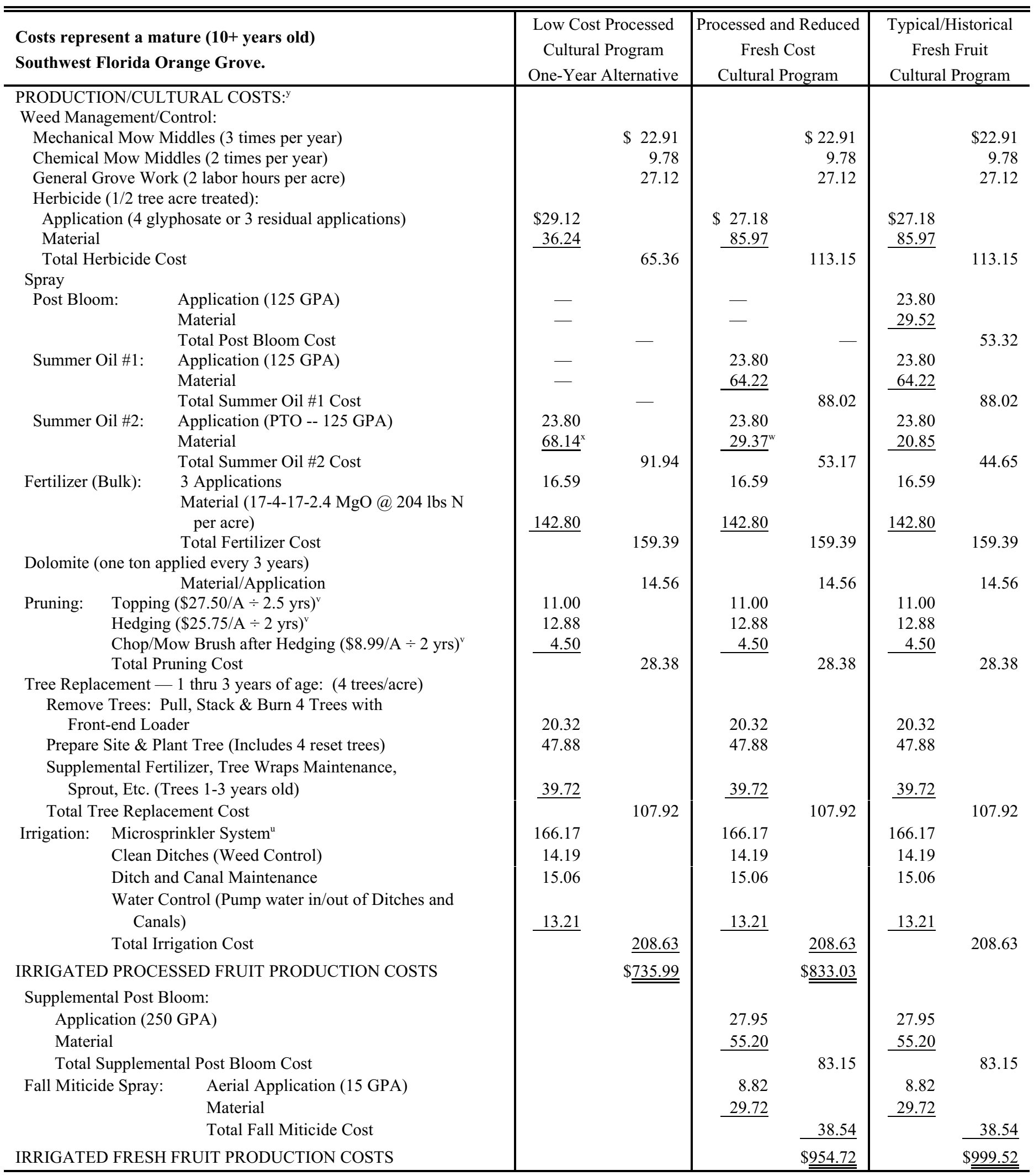

${ }^{z}$ The listed estimated comparative costs are for the example grove situation described in the Economic Information Report Series entitled:

"Budgeting Costs and Returns for Southwest Florida Citrus Production" and may not represent your particular grove situation in Southwest Florida. 
Table 12-A.--A listing of estimated comparative Southwest Florida citrus production costs per acre for grapefruit, 2004-05

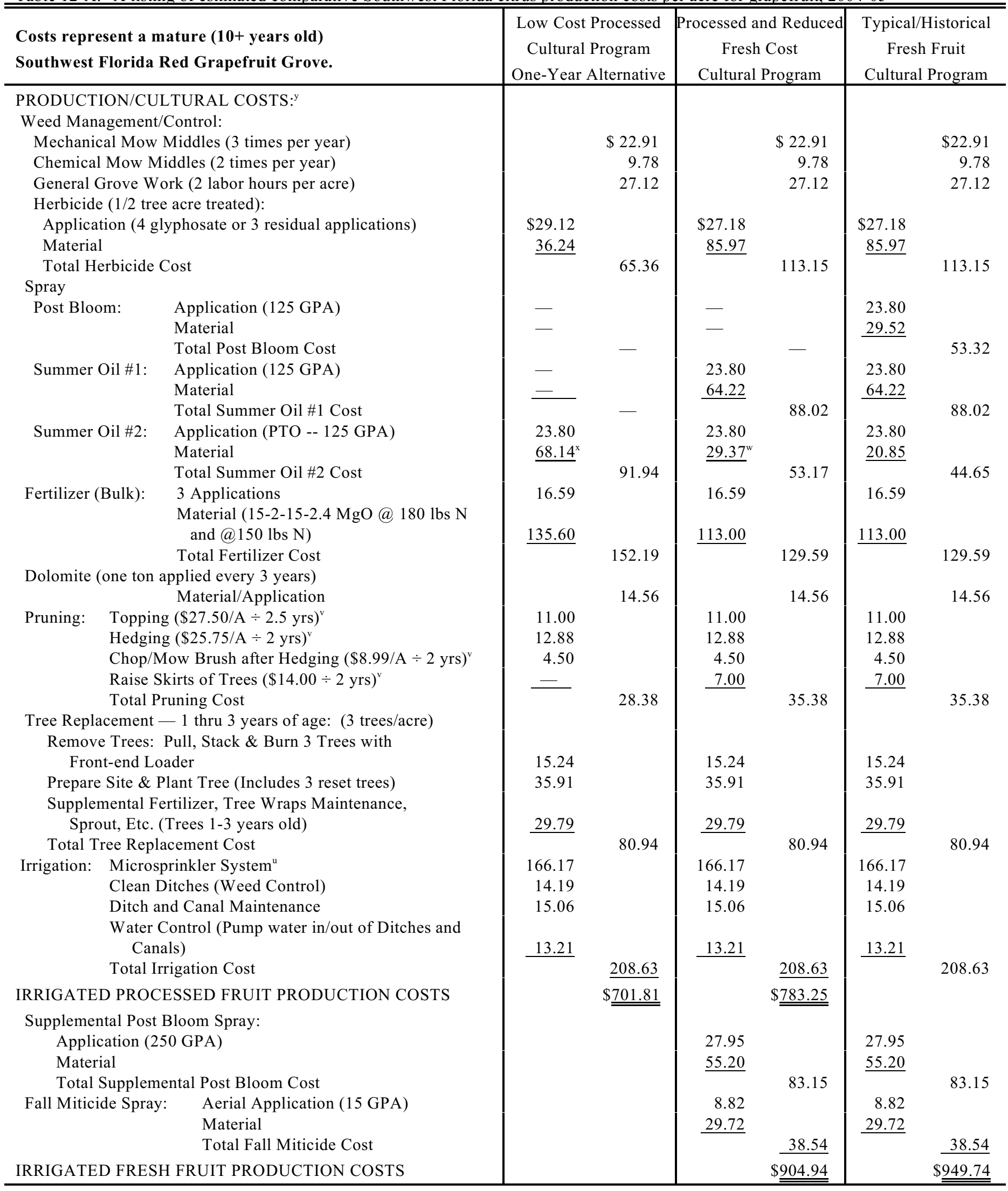

${ }^{\mathrm{z}}$ The listed estimated comparative costs are for the example grove situation described in the Economic Information Report Series entitled: "Budgeting Costs and Returns for Southwest Florida Citrus Production" and may not represent your particular grove situation in Southwest Florida. 
Table 13-A.--Estimated cost of planting and maintaining a reset citrus tree through three years of age, Southwest Florida area, August 2005

\begin{tabular}{|c|c|c|c|c|c|}
\hline & \multicolumn{5}{|c|}{ Resets/Replacement Trees Per Acre } \\
\hline & $1-2$ & $3-5$ & $6-10$ & $11-25$ & $26+$ \\
\hline & \multicolumn{5}{|c|}{ 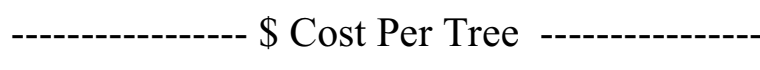 } \\
\hline Tree Removal & 6.67 & 5.34 & 4.45 & 3.56 & 2.67 \\
\hline \multicolumn{6}{|l|}{ Plant ResetTree } \\
\hline Tree Cost (Container Tree) & 4.50 & 4.50 & 4.35 & 4.35 & 4.35 \\
\hline Plant Tree and First Watering (Custom Charge) & 2.93 & 2.55 & 2.17 & 1.84 & 1.57 \\
\hline Total Planting Costs & 7.43 & 7.05 & 6.52 & 6.19 & 5.92 \\
\hline
\end{tabular}

$\underline{\text { Site Preparation }}^{\mathrm{a}}$

Rotovate

$\begin{array}{lllll}2.65 & 2.31 & 1.96 & 1.67 & 1.42 \\ \underline{3.00} & \underline{2.61} & \underline{2.22} & \underline{1.89} & \underline{1.60} \\ 5.65 & 4.92 & 4.18 & 3.56 & 3.02\end{array}$

Total Planting and Site Preparation Costs

$\begin{array}{lllll}13.08 & 11.97 & 10.70 & 9.75 & 8.94\end{array}$

\begin{tabular}{|c|c|c|c|c|c|c|}
\hline Supplemental Maintenance & Year \#1 & 4.13 & 3.82 & 3.59 & 3.39 & 3.19 \\
\hline (Trees 1-3 years old) & Year \#2 & 3.79 & 3.39 & 2.96 & 2.59 & 2.27 \\
\hline (Fertilizer, Tree Wraps, Sprout, etc.) & Year \#3 & $\underline{3.07}$ & $\underline{2.73}$ & 2.34 & 2.01 & 1.73 \\
\hline \multicolumn{2}{|c|}{ Total Supplemental Maintenance Costs } & 10.99 & 9.94 & 8.89 & 7.99 & 7.19 \\
\hline \multicolumn{2}{|l|}{ Summary of Tree Replacement Costs } & 1 & 3 & 6 & 6 & 6 \\
\hline \multicolumn{2}{|l|}{ Tree Removal Costs } & 6.67 & 5.34 & 4.45 & 3.56 & 2.67 \\
\hline \multicolumn{2}{|l|}{ Planting and Tree Removal Costs } & 13.08 & 11.97 & 10.70 & 9.75 & 8.94 \\
\hline \multicolumn{2}{|c|}{ Supplemental Maintenance Costs (Years 1 thru 3) } & $\underline{10.99}$ & $\underline{9.93}$ & $\underline{8.90}$ & $\underline{7.99}$ & $\underline{7.19}$ \\
\hline \multicolumn{2}{|l|}{ Total Three-Year Cumulative Costs } & $\underline{\underline{30.74}}$ & $\underline{\underline{27.24}}$ & $\underline{\underline{24.05}}$ & $\underline{\underline{21.30}}$ & $\underline{\underline{18.80}}$ \\
\hline
\end{tabular}

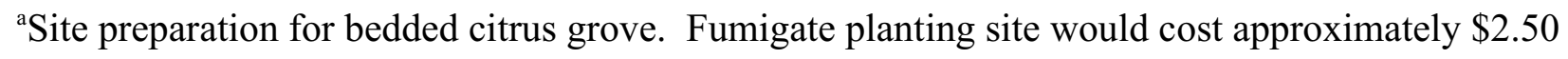
per tree.

Source: Ronald P. Muraro, Farm Management Economist, CREC, Lake Alfred, FL, August 2005. 
Table 14-A.-- Estimated average picking, roadsiding and hauling charges for Florida citrus, 2004-05

\begin{tabular}{|c|c|c|c|c|c|c|}
\hline & \multicolumn{3}{|c|}{ Fresh Fruit } & \multicolumn{3}{|c|}{ Processed Fruit } \\
\hline & \multicolumn{2}{|c|}{ Range } & Average & \multicolumn{2}{|r|}{ Range } & Average \\
\hline & \multicolumn{2}{|c|}{$\$ /$ Box } & $\$ /$ Box & \multicolumn{2}{|r|}{$\$ /$ Box } & $\$ /$ Box \\
\hline \multicolumn{7}{|l|}{ Picking Charges: } \\
\hline Early and Mid-Season Oranges & 0.70 & $-\quad 1.75$ & 0.954 & 0.65 & $-\quad 1.05$ & 0.829 \\
\hline Valencia Oranges & 0.70 & $-\quad 1.25$ & 0.938 & 0.65 & $-\quad 1.25$ & 0.870 \\
\hline Pink/Red Grapefruit & 0.60 & $-\quad 1.25$ & 0.739 & 0.55 & $-\quad 1.25$ & 0.669 \\
\hline White/Marsh Grapefruit & 0.60 & -1.25 & 0.744 & 0.55 & -1.25 & 0.667 \\
\hline Temples/Tangelos & 0.85 & $-\quad 1.65$ & 1.163 & 0.80 & $-\quad 1.50$ & 1.043 \\
\hline Tangerines & 1.25 & -2.00 & 1.529 & 1.00 & $-\quad 1.70$ & 1.204 \\
\hline \multirow[t]{4}{*}{ Add for Spot Picking } & 0.10 & $-\quad 0.50$ & 0.314 & & - & - \\
\hline & \multicolumn{3}{|c|}{ Fresh Fruit } & \multicolumn{3}{|c|}{ Processed Fruit } \\
\hline & \multicolumn{2}{|c|}{ Range } & Average & \multicolumn{2}{|r|}{ Range } & Average \\
\hline & \multicolumn{2}{|c|}{$\$ /$ Box } & $\$ /$ Box & \multicolumn{2}{|r|}{$\$ /$ Box } & $\$ /$ Box \\
\hline \multicolumn{7}{|l|}{ Roadsiding Charges: } \\
\hline Early and Mid-Season Oranges & 0.60 & $-\quad 1.15$ & 0.895 & 0.65 & $-\quad 1.17$ & 0.817 \\
\hline Valencia Oranges & 0.67 & $-\quad 1.12$ & 0.899 & 0.65 & $-\quad 1.17$ & 0.836 \\
\hline Pink/Red Grapefruit & 0.65 & $-\quad 1.03$ & 0.840 & 0.65 & $-\quad 1.20$ & 0.796 \\
\hline White/Marsh Grapefruit & 0.65 & $-\quad 1.03$ & 0.854 & 0.65 & $-\quad 1.20$ & 0.789 \\
\hline Temples/Tangelos & 0.70 & $-\quad 1.35$ & 1.003 & 0.75 & $-\quad 1.23$ & 0.890 \\
\hline \multirow[t]{4}{*}{ Tangerines } & 0.75 & $-\quad 1.35$ & 1.095 & 0.85 & $-\quad 1.70$ & 1.054 \\
\hline & \multicolumn{3}{|c|}{ Fresh Fruit } & \multicolumn{3}{|c|}{ Processed Fruit } \\
\hline & \multicolumn{3}{|c|}{ All Varieties } & \multicolumn{3}{|c|}{ All Varieties } \\
\hline & \multicolumn{3}{|c|}{$\$ /$ Box } & \multicolumn{3}{|c|}{$\$ /$ Box } \\
\hline \multicolumn{7}{|l|}{ Hauling Charges: } \\
\hline 0 - 30 miles & \multicolumn{3}{|c|}{0.417} & \multicolumn{3}{|c|}{0.393} \\
\hline $31-50$ miles & \multicolumn{3}{|c|}{0.512} & \multicolumn{3}{|c|}{0.464} \\
\hline $51-80$ miles & \multicolumn{3}{|c|}{0.573} & \multicolumn{3}{|c|}{0.515} \\
\hline $81-100$ miles & \multicolumn{3}{|c|}{0.640} & \multicolumn{3}{|c|}{0.632} \\
\hline $100+$ miles & & $0.74 c$ & & & 0.72 & \\
\hline
\end{tabular}


Table 15-A.--Estimated Average Packing Charges for Florida Citrus, 2004-05

\begin{tabular}{|c|c|c|c|c|c|}
\hline & $\begin{array}{l}\text { Domestic } \\
\text { Grapefruit }\end{array}$ & $\begin{array}{c}\text { Export } \\
\text { Grapefruit }\end{array}$ & Oranges & $\begin{array}{l}\text { Temples/ } \\
\text { Tangelos }\end{array}$ & Tangerines \\
\hline & ------ & 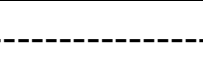 & \$/Carton & ------------. & ---------- \\
\hline \multirow[t]{2}{*}{ Total Packing Charge $^{\mathrm{b}}$} & 3.835 & 4.245 & 4.192 & 4.495 & 5.056 \\
\hline & & & $\$ /$ Box & --------- & ------- \\
\hline Drenching Charge & 0.178 & 0.178 & 0.186 & 0.186 & 0.186 \\
\hline $\begin{array}{l}\text { Packinghouse Elimination } \\
\text { Charges }\end{array}$ & 0.594 & 0.594 & 0.571 & 0.571 & 0.571 \\
\hline $\begin{array}{l}\text { Hauling Charges for } \\
\text { Eliminations }\end{array}$ & 0.425 & 0.425 & 0.410 & 0.410 & 0.410 \\
\hline
\end{tabular}

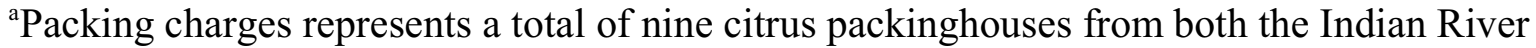
and Interior production regions.

${ }^{\mathrm{b}}$ Total Packing Charge includes the following items:

1. Materials including mesh/plastic bags, labels/Price Lookup Codes (PLUs), etc.

2. Includes supervisor/foreman labor, grading, palletizing, shipping and general labor. Includes payroll taxes, workers' compensation, ground insurance, etc.

3. Other direct packing costs include: fruit treating; power, lights and water; repairs maintenance; miscellaneous supplies; etc.

4. Indirect packing costs include such items as: insurance-fire and casualty; taxes and licenses; depreciation and rent.

5. General and Administrative (G\&A) costs include: office personnel (payroll taxes, w/comp); packinghouse and general manager; office supplies; telephone; etc.

6. Selling Expenses which include sales salaries, travel, telephone and telegraph and brokerage fees.

7. Special assessments include such items as: advertising taxes; inspection fees; a Florida Citrus Packers tax; and a Citrus Administrative Committee (CAC) tax.

SOURCE: Ronald P. Muraro, University of Florida-IFAS, Citrus Research and Education Center, Lake Alfred, FL, September 2005. 
Table 16-A.--Historic prices ${ }^{\mathrm{a}}$ for selected citrus varieties

\begin{tabular}{|c|c|c|c|c|c|c|c|}
\hline \multirow[b]{3}{*}{ Crop year } & \multicolumn{7}{|c|}{ Variety } \\
\hline & \multirow{2}{*}{$\begin{array}{c}\text { Early }^{\mathrm{b}} \text { and } \\
\text { mid }^{\mathrm{c}} \text {-season } \\
\text { oranges }\end{array}$} & \multirow[b]{2}{*}{$\begin{array}{c}\text { Late season } \\
\text { oranges }^{\mathrm{d}}\end{array}$} & \multirow[b]{2}{*}{$\begin{array}{l}\text { Temple } \\
\text { oranges }\end{array}$} & \multirow[b]{2}{*}{$\begin{array}{c}\text { All } \\
\text { Tangerines } \\
\end{array}$} & \multirow[b]{2}{*}{ Tangelos } & \multicolumn{2}{|c|}{ Seedless grapefruit ${ }^{\mathrm{e}}$} \\
\hline & & & & & & (white) & (colored) \\
\hline $1961-62$ & $\$ 1.93$ & $\$ 1.81$ & $\$ 2.17$ & $\$ 2.04$ & $\$ 3.36$ & $\$ 0.68$ & $\$ 0.86$ \\
\hline $1962-63$ & 2.17 & 3.50 & 3.09 & 3.02 & 4.66 & 1.29 & 1.81 \\
\hline $1963-64$ & 4.43 & 4.45 & 4.45 & 3.18 & 4.83 & 2.24 & 2.54 \\
\hline $1964-65$ & 2.57 & 2.28 & 2.77 & 2.68 & 4.00 & 1.51 & 1.82 \\
\hline $1965-66$ & 1.44 & 1.79 & 1.80 & 2.14 & 2.85 & 1.39 & 1.64 \\
\hline $1966-67$ & 0.81 & 1.08 & 0.88 & 1.06 & 1.64 & 0.73 & 0.94 \\
\hline $1967-68$ & 1.86 & 2.28 & 2.79 & 4.29 & 3.22 & 2.05 & 2.48 \\
\hline $1968-69$ & 1.56 & 1.83 & 2.22 & 2.55 & 2.47 & 0.98 & 1.15 \\
\hline $1969-70$ & 1.15 & 1.13 & 1.47 & 2.23 & 1.13 & 1.72 & 1.92 \\
\hline $1970-71$ & 1.10 & 1.91 & 1.91 & 1.88 & 1.04 & 1.89 & 2.15 \\
\hline $1971-72$ & 1.98 & 2.11 & 1.95 & 2.97 & 1.69 & 2.27 & 2.69 \\
\hline $1972-73$ & 1.43 & 1.71 & 1.95 & 2.37 & 1.39 & 2.06 & 2.53 \\
\hline $1973-74$ & 1.38 & 1.59 & 1.64 & 2.82 & 1.25 & 1.58 & 2.12 \\
\hline $1974-75$ & 1.46 & 1.82 & 1.68 & 3.05 & 1.45 & 1.55 & 2.59 \\
\hline $1975-76$ & 1.69 & 1.88 & 1.79 & 3.02 & 1.42 & 1.29 & 2.23 \\
\hline $1976-77$ & 1.89 & 2.63 & 2.16 & 3.29 & 1.42 & 1.49 & 2.04 \\
\hline $1977-78$ & 3.90 & 4.40 & 3.92 & 4.79 & 3.29 & 1.47 & 2.09 \\
\hline $1978-79$ & 4.44 & 4.95 & 4.89 & 4.99 & 3.90 & 2.21 & 3.13 \\
\hline $1979-80$ & 3.59 & 3.89 & 2.89 & 4.25 & 2.87 & 3.12 & 3.80 \\
\hline $1980-81$ & 3.67 & 4.63 & 4.21 & 5.45 & 3.92 & 3.46 & 4.22 \\
\hline $1981-82$ & 4.27 & 4.29 & 4.01 & 6.23 & 3.58 & 1.92 & 2.80 \\
\hline $1982-83$ & 4.88 & 5.41 & 3.99 & 7.57 & 4.37 & 1.51 & 3.20 \\
\hline $1983-84$ & 5.09 & 6.72 & 5.34 & 5.93 & 4.28 & 2.08 & 4.05 \\
\hline $1984-85$ & 7.30 & 6.88 & 5.59 & 15.91 & 7.08 & 3.02 & 4.84 \\
\hline $1985-86$ & 3.92 & 3.97 & 3.01 & 12.69 & 4.06 & 3.56 & 4.98 \\
\hline $1986-87$ & 4.56 & 6.02 & 3.60 & 10.92 & 3.72 & 4.45 & 5.80 \\
\hline $1987-88$ & 6.72 & 8.73 & 5.69 & 12.99 & 5.58 & 5.35 & 5.93 \\
\hline $1988-89$ & 6.63 & 8.41 & 5.46 & 12.64 & 6.31 & 4.33 & 4.71 \\
\hline $1989-90$ & 6.01 & 6.53 & 5.64 & 15.28 & 5.10 & 5.21 & 6.30 \\
\hline $1990-91$ & 5.38 & 6.58 & 6.31 & 17.10 & 6.11 & 4.59 & 6.85 \\
\hline $1991-92$ & 5.44 & 6.65 & 6.51 & 18.00 & 7.16 & 6.46 & 6.87 \\
\hline $1992-93$ & 3.23 & 3.88 & 2.99 & 13.75 & 3.31 & 2.22 & 3.11 \\
\hline $1993-94$ & 3.76 & 4.61 & 2.73 & 9.83 & 2.38 & 3.23 & 3.38 \\
\hline $1994-95$ & 3.25 & 4.41 & 3.47 & 11.98 & 2.64 & 2.58 & 1.66 \\
\hline $1995-96$ & 3.62 & 5.57 & 4.44 & 12.59 & 3.63 & 2.14 & 1.77 \\
\hline $1996-97$ & 3.18 & 4.07 & 3.22 & 7.99 & 2.19 & 1.12 & 1.91 \\
\hline $1997-98$ & 2.81 & 4.88 & 3.07 & 8.49 & 1.66 & 0.93 & 1.50 \\
\hline 1998-99 & 4.35 & 5.58 & 5.12 & 12.07 & 4.53 & 1.95 & 2.65 \\
\hline 1999-00 & 3.19 & 4.33 & 2.55 & 6.67 & 2.52 & 3.87 & 3.36 \\
\hline 2000-01 & 2.60 & 4.02 & 2.05 & 6.40 & 1.27 & 2.07 & 2.28 \\
\hline 2001-02 & 2.88 & 4.20 & 2.19 & 7.81 & 2.47 & 1.96 & 2.54 \\
\hline $2002-03$ & 2.62 & 3.85 & 2.01 & 8.40 & 2.60 & 1.59 & 2.79 \\
\hline $2003-04$ & 2.20 & 3.64 & 1.07 & 7.46 & 7.48 & 1.88 & 3.28 \\
\hline $2004-05^{\mathrm{f}}$ & 2.56 & 4.34 & 2.48 & 12.02 & 2.45 & 11.95 & 13.65 \\
\hline
\end{tabular}

${ }^{\mathrm{a} O n-t r e e ~ a v e r a g e ~ p r i c e ~ p e r ~ b o x ~(1-3 / 5 ~ b u s h e l ~ b o x ~ e q u i v a l e n t) ~ f o r ~ a l l ~ m e t h o d s ~ o f ~ s a l e ~ m i n u s ~ p i c k ~ a n d ~ h a u l ~ c h a r g e s . ~}$

${ }^{b}$ Navel and Hamlin $\quad{ }^{c}$ Parson Brown and Pineapple $\quad{ }^{d}$ Valencia $\quad{ }^{e}$ Marsh (white) or pink Preliminary

Source: Florida Agricultural Statistics Service. 
Table 17-A.--Debt which can be supported per $\$ 1,000.00$ annual payment capacity

\begin{tabular}{|c|c|c|c|c|c|c|c|c|c|c|c|c|c|c|c|}
\hline \multirow{2}{*}{$\begin{array}{c}\text { Loan } \\
\text { term } \\
\text { (years) }\end{array}$} & \multicolumn{15}{|c|}{ Interest rate paid on the loan } \\
\hline & $8.0 \%$ & $8.5 \%$ & $9.0 \%$ & $9.5 \%$ & $10.0 \%$ & $10.5 \%$ & $11.0 \%$ & $11.5 \%$ & $12.0 \%$ & $12.5 \%$ & $13.0 \%$ & $13.5 \%$ & $14.0 \%$ & $14.5 \%$ & $15.0 \%$ \\
\hline 1 & 926 & 922 & 917 & 913 & 909 & 905 & 901 & 897 & 893 & 889 & 885 & 881 & 877 & 873 & 870 \\
\hline 2 & 1,783 & 1,771 & 1,759 & 1,747 & 1,754 & 1,724 & 1,713 & 1,701 & 1,690 & 1,679 & 1,668 & 1,657 & 1,647 & 1,636 & 1,626 \\
\hline 3 & 2,577 & 2,554 & 2,531 & 2,509 & 2,487 & 2,465 & 2,444 & 2,423 & 2,402 & 2,381 & 2,361 & 2,341 & 2,322 & 2,302 & 2,283 \\
\hline 4 & 3,312 & 3,276 & 3,240 & 3,204 & 3,170 & 3,136 & 3,102 & 3,070 & 3,037 & 3,006 & 2,974 & 2,944 & 2,914 & 2,884 & 2,855 \\
\hline 5 & 3,993 & 3,941 & 3,890 & 3,840 & 3,791 & 3,743 & 3,696 & 3,650 & 3,605 & 3,561 & 3,517 & 3,475 & 3,433 & 3,392 & 3,352 \\
\hline 6 & 4,623 & 4,554 & 4,486 & 4,420 & 4,355 & 4,292 & 4,230 & 4,170 & 4,111 & 4,054 & 3,998 & 3,942 & 3,889 & 3,836 & 3,784 \\
\hline 7 & 5,206 & 5,119 & 5,033 & 4,950 & 4,868 & 4,789 & 4,712 & 4,640 & 4,564 & 4,492 & 4,423 & 4,355 & 4,288 & 4,224 & 4,160 \\
\hline 8 & 5,747 & 5,639 & 5,535 & 5,433 & 5,335 & 5,239 & 5,146 & 5,056 & 4,968 & 4,882 & 4,799 & 4,718 & 4,639 & 4,562 & 4,487 \\
\hline 9 & 6,247 & 6,119 & 5,995 & 5,875 & 5,759 & 5,646 & 5,537 & 5,431 & 5,328 & 5,228 & 5,132 & 5,038 & 4,946 & 4,858 & 4,772 \\
\hline 10 & 6,710 & 6,561 & 6,418 & 6,279 & 6,145 & 6,015 & 5,889 & 5,768 & 5,650 & 5,536 & 5,426 & 5,319 & 5,216 & 5,116 & 5,019 \\
\hline 11 & 7,139 & 6,969 & 6,805 & 6,647 & 6,495 & 6,348 & 6,207 & 6,070 & 5,938 & 5,810 & 5,687 & 5,568 & 5,453 & 5,341 & 5,234 \\
\hline 12 & 7,536 & 7,345 & 7,161 & 6,984 & 6,814 & 6,650 & 6,492 & 6,341 & 6,194 & 6,054 & 5,918 & 5,787 & 5,660 & 5,538 & 5,421 \\
\hline 13 & 7,904 & 7,691 & 7,487 & 7,291 & 7,103 & 6,923 & 6,750 & 6,583 & 6,424 & 6,270 & 6,122 & 5,979 & 5,842 & 5,710 & 5,583 \\
\hline 14 & 8,244 & 8,010 & 7,786 & 7,572 & 7,367 & 7,170 & 6,982 & 6,801 & 6,628 & 6,462 & 6,302 & 6,149 & 6,002 & 5,861 & 5,724 \\
\hline$\underline{15}$ & 8,559 & 8,304 & 8,061 & 7,828 & 7,606 & 7,394 & 7,191 & ${\underline{6,997^{\mathrm{a}}}}$ & 6,811 & 6,633 & 6,462 & 6,299 & 6,142 & 5,992 & 5,847 \\
\hline 16 & 8,851 & 8,576 & 8,313 & 8,062 & 7,824 & 7,596 & 7,379 & 7,172 & 6,974 & 6,785 & 6,604 & 6,431 & 6,265 & 6,106 & 5,954 \\
\hline 17 & 9,122 & 8,825 & 8,543 & 8,276 & 8,022 & 7,779 & 7,549 & 7,329 & 7,119 & 6,920 & 6,729 & 6,547 & 6,373 & 6,207 & 6,048 \\
\hline 18 & 9,372 & 9,056 & 8,756 & 8,471 & 8,201 & 7,945 & 7,702 & 7,470 & 7,250 & 7,040 & 6,840 & 6,649 & 6,467 & 6,294 & 6,128 \\
\hline 19 & 9,603 & 9,268 & 8,950 & 8,650 & 8,365 & 8,095 & 7,839 & 7,596 & 7,366 & 7,146 & 6,938 & 6,739 & 6,551 & 6,370 & 6,198 \\
\hline$\underline{20}$ & 9,818 & 9,463 & 9,129 & 8,812 & 8,514 & 8,231 & 7,963 & ${\underline{7,710^{\mathrm{a}}}}^{\mathrm{a}}$ & 7,469 & 7,241 & 7,025 & 6,819 & 6,623 & 6,437 & 6,259 \\
\hline 25 & 10,675 & 10,234 & 9,823 & 9,438 & 9,077 & 8,739 & 8,422 & 8,123 & 7,843 & 7,579 & 7,330 & 7,095 & 6,873 & 6,663 & 6,464 \\
\hline 30 & 11,258 & 10,747 & 10,274 & 9,835 & 9,427 & 9,047 & 8,868 & 8,364 & 8,055 & 7,766 & 7,496 & 7,242 & 7,003 & 6,778 & 6,566 \\
\hline 35 & 11,655 & 11,088 & 10,567 & 10,087 & 9,644 & 9,234 & 8,855 & 8,503 & 8,175 & 7,870 & 7,586 & 7,320 & 7,070 & 6,836 & 6,617 \\
\hline 40 & 11,925 & 11,315 & 10,757 & 10,247 & 9,779 & 9,348 & 8,951 & 8,587 & 8,244 & 7,928 & 7,634 & 7,361 & 7,105 & 6,866 & 6,642 \\
\hline
\end{tabular}

${ }^{a}$ Example. Assumes a $\$ 10,000$ after tax income at $11.5 \%$ interest rate and a 15 -year term mortgage, the total debt which can be supported is $\$ 69,970$ $(\$ 6,997 \times 10)$. At $11.5 \%$ interest rate and a 20 -year term mortgage, the total debt which can be supported is $\$ 77,100(\$ 7,710 \times 10)$. 